\title{
Synthesis of Methyl 4,6-Di-O-ethyl- $\alpha$-D-glucopyranoside-Based Azacrown Ethers and Their Effects in Asymmetric Reactions
}

\author{
István Orbán (D), Bertalan Varga, Péter Bagi (D), László Hegedús (D), Péter Bakó and Zsolt Rapi *(D) \\ Department of Organic Chemistry and Technology, Budapest University of Technology and Economics, \\ H-1111 Budapest, Hungary; istvan.orban@edu.bme.hu (I.O.); varga.bertalan@edu.bme.hu (B.V.); \\ bagi.peter@vbk.bme.hu (P.B.); hegedus.laszlo@vbk.bme.hu (L.H.); bako.peter@vbk.bme.hu (P.B.) \\ * Correspondence: rapi.zsolt@vbk.bme.hu
}

Citation: Orbán, I.; Varga, B.; Bagi, P.; Hegedûs, L.; Bakó, P.; Rapi, Z.

Synthesis of Methyl 4,6-Di-O-ethyl- $\alpha$ D-glucopyranoside-Based Azacrown Ethers and Their Effects in Asymmetric Reactions. Molecules 2021, 26, 4668. https://doi.org/ $10.3390 /$ molecules 26154668

Academic Editor: Chuanfeng Chen

Received: 4 July 2021

Accepted: 29 July 2021

Published: 2 August 2021

Publisher's Note: MDPI stays neutral with regard to jurisdictional claims in published maps and institutional affiliations.

Copyright: (c) 2021 by the authors. Licensee MDPI, Basel, Switzerland. This article is an open access article distributed under the terms and conditions of the Creative Commons Attribution (CC BY) license (https:// creativecommons.org/licenses/by/ $4.0 /)$.
Abstract: Carbohydrate-based crown ethers have been reported to be able to generate asymmetric induction in certain reactions. Previously, it was proved that the monosaccharide unit, the anomeric substituent, and the sidearm could influence the catalytic activity of the monoaza-15-crown5 macrocycles derived from sugars. In order to gain information about the effect of the flexibility, 4,6-di-O-ethyl-glucoside-based crown compounds were synthesized, and their efficiency was compared to the 4,6-O-benzylidene analogues. It was found that the absence of the two-ring annulation has a negative effect on the enantioselectivity in liquid-liquid two-phase reactions: in the Darzens condensation of 2-chloroacetophenone and in the epoxidation of chalcone. The same trend was observed in the solid-liquid phase Michael addition of diethyl acetamidomalonate. Surprisingly, in the solid-liquid phase cyclopropanation of benzylidenemalononitrile, one of the new catalysts was highly enantioselective ( $99 \%$ ee).

Keywords: crown ether; carbohydrates; enantioselectivity; asymmetric synthesis

\section{Introduction}

Catalysis is one of the key technologies for achieving sustainable chemistry. Organocatalysis is a recently developed method that is a particularly favorite field in enantioselective synthesis [1-4]. This technique uses metal-free compounds that can catalyze organic reactions. Asymmetric organocatalysis applies chiral organic molecules to access enantioenriched products. Within the extremely fast-growing field of organocatalysis, catalysts derived from chincona alkaloids have been successfully used in enantioselective syntheses [5-14]. The quaternization of the nitrogen of the quinuclidine unit in the cinchona alkaloids results in the formation of organocatalysts, which can also act as phase transfer catalysts.

Phase transfer catalysis (PTC), a special case of organocatalysis, is a useful technique in organic syntheses that has long been recognized as a simple method using mild reaction conditions, inexpensive and environmentally benign reagents, and solvents. PTC offers the possibility of realizing preparations on a larger scale, as well $[15,16]$. Since the 1990s, asymmetric PTC has emerged as a topic of scientific interest. Consequently, different chiral catalysts derived from cinchona alkaloids, amino acids, and 1,1'-bi-2-naphthol (BINOL) alongside macrocyclic compounds have been synthesized and applied [17-35].

Chiral crown ethers applied as phase transfer catalysts can be used in asymmetric syntheses. Cram and his coworkers have published the first enantioselective phase-transfer reaction using a chiral crown ether catalyst derived from synthetic 1,1'-bi-2-naphthol [36]. The development of chiral catalysts from naturally occurring and cheap enantiopure compounds (e.g., from carbohydrates) is one of the challenges of green chemistry.

Previously, it has been proven that crown ethers containing a monosaccharide unit annulated to the macro ring can generate asymmetric induction as chiral phase transfer catalysts in certain reactions [37-39]. Crown ethers incorporating sugar moieties have been 
synthesized from various carbohydrates, e.g., from D-glucose [40,41], D-galactose [42,43], Dmannose [44,45], D-altrose [46], D- and L-xylose [47,48], L-arabinose [49], D-mannitol [50-52] or L-threitol [53]. Thus, a broad scale of carbohydrate-based macrocycles with diverse chirality are available. A part of these crown ethers may be used as chiral phase transfer catalysts in asymmetric syntheses [54,55].

While the structure-activity relationship has been investigated in our research group, it was found that the most active catalysts are the monoaza-15-crown-5-type lariat ethers containing a monosaccharide unit. There are other moieties that affect the catalytic activity of the azacrown compounds in addition to the carbohydrate unit, such as the sidearm on the nitrogen [56], the substituent and the configuration of the anomeric center [57-59], and the acetal group in the 4,6-position of the monosaccharide [60,61].

The bicyclic acetal structure provides rigidity to the carbohydrate moiety. Thus, the conformational changes require more energy, which may have a crucial role in enantioselectivity. Investigation of azacrown ethers bearing more flexible butyl substituents in the 4,6 position of the glucose molecule revealed that the effects of the individual moieties are not independent. Application of a crown catalyst having larger conformational freedom can lead to higher enantioselectivity depending on the structure of the sidearm [62].

Herein, we report the synthesis of a few methyl 4,6-di-O-ethyl-glucoside-based crown ethers bearing different side chains on the nitrogen. The most effective sidearms to date, hydroxypropyl (1a), methoxypropyl (1b), and 2-methoxyphenylethyl groups (1c) were incorporated into the new catalysts (Figure 1). Ethyl groups provide greater flexibility to crown compounds 1a-c; however, they do not excessively increase lipophilicity. The efficiency of the macrocycles 1a-c was investigated in different asymmetric reactions, and the results were compared with those obtained with their benzylidene analogues $2 \mathbf{a}-\mathbf{c}$ (Figure 1) to establish correlations between the effect and the structure. Crown ether 2a was previously investigated in all the model reactions applied for the catalyst testing [63-66].<smiles>[R]CN1CCOCCO[C@H](OCC)[C@H](OCCOCC)[C@@H](OC)O1</smiles>

$$
\begin{aligned}
\text { 1a } \mathrm{R} & =\left(\mathrm{CH}_{2}\right)_{3} \mathrm{OH} \\
\text { 1b } \mathrm{R} & =\left(\mathrm{CH}_{2}\right)_{3} \mathrm{OCH} \\
\text { 1c } \mathrm{R} & =\left(\mathrm{CH}_{2}\right)_{2}\left(2-\mathrm{CH}_{3} \mathrm{O}-\mathrm{C}_{6} \mathrm{H}_{4}\right)
\end{aligned}
$$<smiles>[R]O[C@H]1O[C@H]2CO[C@@H](c3ccccc3)O[C@H]2[C@H](OCCOCCN(CCO)CCOCCO)[C@H]1O</smiles>

$$
\begin{aligned}
& \text { 2a R }=\left(\mathrm{CH}_{2}\right)_{3} \mathrm{OH} \\
& \text { 2b R }=\left(\mathrm{CH}_{2}\right)_{3} \mathrm{OCH} \mathrm{H}_{3} \\
& \text { 2c R }=\left(\mathrm{CH}_{2}\right)_{2}\left(2-\mathrm{CH}_{3} \mathrm{O}-\mathrm{C}_{6} \mathrm{H}_{4}\right)
\end{aligned}
$$

Figure 1. Crown ethers derived from 4,6-di-O-ethyl-glucoside (1a-c) and their 4,6-O-benzylidene analogues $(\mathbf{2 a}-\mathbf{c})$.

\section{Results and Discussion}

\subsection{Synthesis of Azacrown Ethers}

According to the literature, the starting material, methyl 4,6-O-benzylidene- $\alpha$-Dglucopyranoside (3), was previously synthesized in our research group [67]. Next, the free 2- and 3-hydroxy groups were benzylated with benzyl bromide using a solid potassium hydroxide base in boiling toluene as stated in a previously reported method (Scheme 1) [68]. Full conversion was achieved after $6 \mathrm{~h}$, and the pure dibenzyl product 4 was obtained after recrystallization from ethanol with a good yield. 


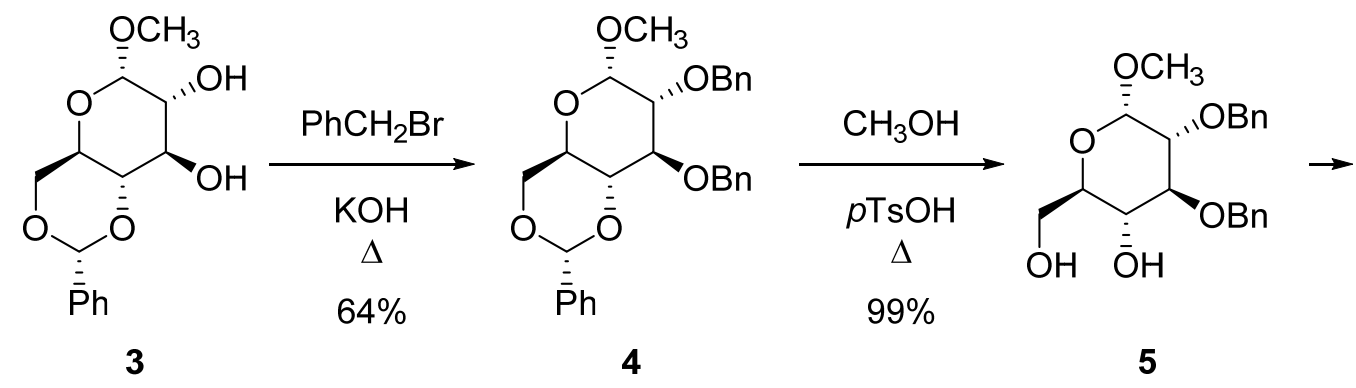<smiles>CCOC[C@H]1O[C@H](OC)[C@@H](O)[C@H](O)[C@H]1COCCO[Mg]</smiles>

Scheme 1. Synthesis of methyl 4,6-di-O-ethyl- $\alpha$-D-glucopyranoside (7) from methyl 4,6-Obenzylidene- $\alpha$-D-glucopyranoside (3).

Subsequently, the benzylidene group of compound 4 was removed by transacetalation. Excess methanol was used as both reagent and solvent in the reaction, and paratoluenesulfonic acid acted as a catalyst (Scheme 1) [69]. Under anhydrous conditions, the benzylidene group is cleaved by the formation of benzaldehyde dimethyl acetal, liberating the 4- and 6-hydroxy groups in derivative 5 . After the workup procedure, the crude product was a colorless syrup, from which fibrous crystals precipitated upon cooling in a refrigerator. The white, fluffy product 5 was obtained in almost quantitative yield after washing with hexane.

Afterward, the free 4- and 6-hydroxy groups of the diol 5 were alkylated with ethyl iodide in the presence of sodium hydride in anhydrous tetrahydrofuran under argon (Scheme 1). To achieve complete conversion, a large excess of both the base and the alkylating agent had to be applied, and a total of $40 \mathrm{~h}$ of boiling was required. The most abundant impurity was the monoalkylated derivative, from which the main product $\mathbf{6}$ was purified by column chromatography.

The last step before the construction of the crown ring was the removal of benzyl groups from the 2- and 3-positions of glucoside 6 . Selective deprotection was carried out by catalytic hydrogenation in an autoclave (15 bar hydrogen pressure) in the presence of $\mathrm{Pd} / \mathrm{C}$ (Scheme 1). The ${ }^{1} \mathrm{H}$ and ${ }^{13} \mathrm{C}$ NMR spectra of the glucose derivative 7 showed the absence of the aromatic and benzylic signals, thus confirming the completion of the reaction.

As described previously by us in many cases, the crown structure was synthesized in three steps [55]. The free vicinal hydroxyl groups of compound 7 were alkylated with bis(2-chloroethyl) ether in a liquid-liquid two-phase system, in which $\mathrm{Bu}_{4} \mathrm{OH}$ was generated from $50 \%$ aq. $\mathrm{NaOH}$ and tetrabutylammonium hydrogensulfate (Scheme 2). After chromatography, bischloro podand 8 was obtained in good yield (72\%). The exchange of chlorine to iodine was performed by reacting bischloro compound 8 with $\mathrm{NaI}$ in dry acetone (Scheme 2). Derivative 9 was isolated without purification in a yield of $84 \%$. Macrocyclization was carried out with 3-aminopropanol, 3-methoxypropylamine, and 2-(2-methoxyphenyl)ethylamine in boiling acetonitrile, applying $\mathrm{Na}_{2} \mathrm{CO}_{3}$ as the base (and to exploit the template effect) to provide azacrown ethers 1a-c in yields of $60-69 \%$ after column chromatography (Scheme 2). 

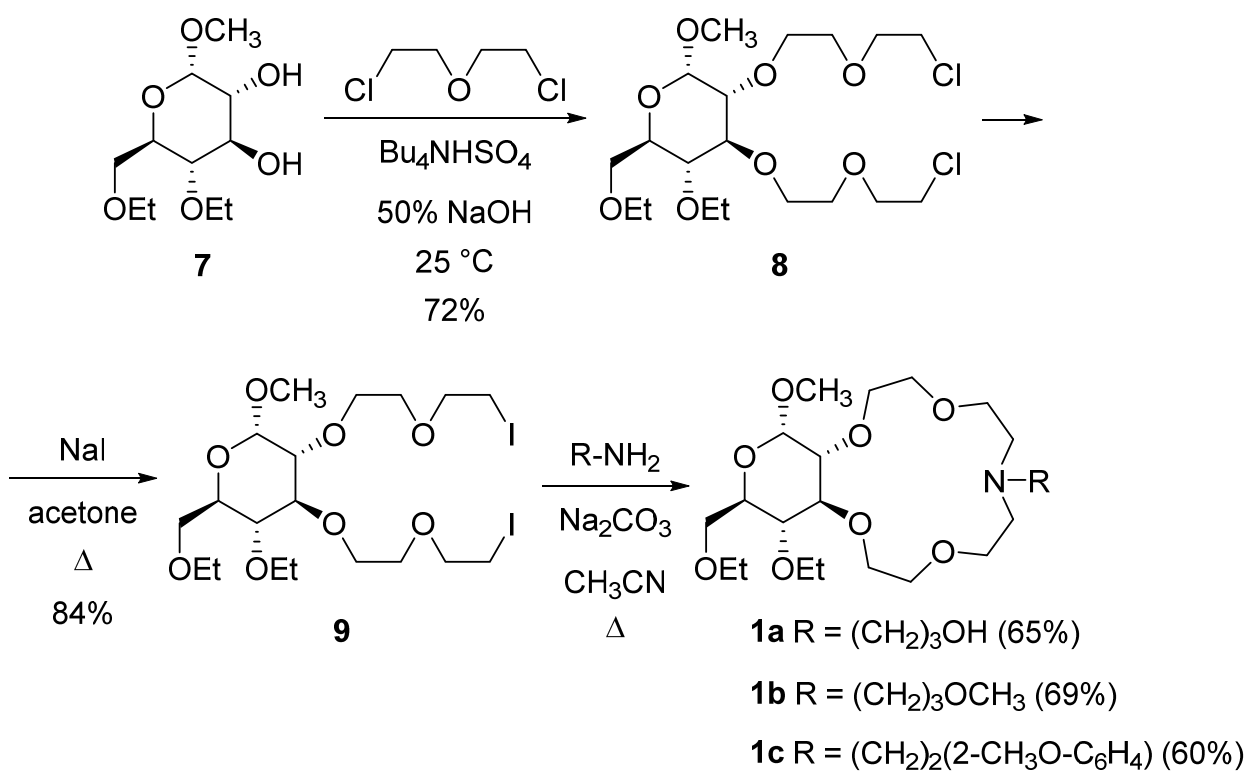

Scheme 2. Preparation of azacrown ethers 1a-c in three steps.

Several attempts were made to prepare catalysts $\mathbf{1 a}-\mathbf{c}$ in a more concise way, starting from crown compounds $\mathbf{2} \mathbf{a}-\mathbf{c}$. Removal of the benzylidene group proceeded easily; however, the alkylation of the crown ethers bearing free $\mathrm{OH}$ groups gave the expected products in relatively low yields $(<10 \%)$. Presumably, side reactions were initiated with the alkylation of the nitrogen, resulting in a mixture of inseparable compounds.

\subsection{Enantioselective Reactions}

Crown ethers 1a-c derived from 4,6-di-O-ethyl-glucopyranoside were tested in two asymmetric liquid-liquid and two solid-liquid phase transfer reactions. The results were compared to the effects of the analogous 4,6-O-benzylidine catalysts $2 \mathbf{a}-\mathbf{c}$. The results of the asymmetric reactions in the presence of crown ether $2 \mathbf{a}$ have already been reported in previous works [63-66]. In all cases, $10 \mathrm{~mol} \%$ of the crown compound (1 or 2) was used. After completion of the reaction, crude products were isolated by preparative thin-layer chromatography (TLC). Chiral HPLC measurements determined the ee values. In each asymmetric reaction, it was always the same enantiomers that were formed in excess.

One of the asymmetric reactions was the base-initiated Darzens condensation of 2-chloroacetophenone (10) and benzaldehyde (11) (Scheme 3). The synthesis resulted in chiral epoxide 12 with complete diastereoselectivity, while a new $\mathrm{C}-\mathrm{C}$ bond was established. The absolute configuration of epoxyketone 12 was previously assigned as $2 R, 3 S[63,70]$. Using chiral crown ethers $\mathbf{1 a}-\mathbf{c}$, full conversion was reached within one hour, as was the case with benzylidene analogues $\mathbf{2 a}-\mathbf{c}$ (Table 1). The same trend was observed for both series of macrocycles. The best enantioselectivity was provided by catalysts $1 \mathbf{a}$ and 2a bearing a hydroxypropyl sidearm (52\% and $62 \%$, Table 1 , entries 1 and 4$)$. When a methoxypropyl group was present, the asymmetric induction decreased to $29 \%$ (1) b) and $21 \%(\mathbf{2 b})$, respectively (Table 1, entries 2 and 5).<smiles>O=C(CCl)c1ccccc1</smiles>

10<smiles>O=Cc1ccccc1</smiles>

11<smiles>O=C(c1ccccc1)[C@H]1O[C@@H]1c1ccccc1</smiles>

12

Scheme 3. Darzens condensation of 2-chloroacetophenone (10) and benzaldehyde (11) in the presence of sugar-based crown ethers ( $\mathbf{1}$ or $\mathbf{2}$ ). 
Table 1. Effect of the crown catalysts ( $\mathbf{1}$ or $\mathbf{2}$ ) in Darzens condensation of 2-chloroacetophenone (10) and benzaldehyde (11).

\begin{tabular}{ccccc}
\hline Entry & Catalyst & Time, & Yield, $\mathbf{~ a ~}$ & ee, $\mathbf{o}$ \\
\hline 1 & $\mathbf{1 a}$ & 1 & 69 & 52 \\
2 & $\mathbf{1 b}$ & 1 & 68 & 29 \\
3 & $\mathbf{1 c}$ & 1 & 66 & 19 \\
4 & $\mathbf{2 a}$ & 1 & 74 & $62^{\mathrm{c}}$ \\
5 & $\mathbf{2 b}$ & 1 & 74 & $21^{\mathrm{c}}$ \\
6 & $\mathbf{2 c}$ & 1 & 38 & 29
\end{tabular}

${ }^{a}$ Isolated by preparative TLC; ${ }^{b}$ Determined by chiral HPLC; ${ }^{c}$ See Ref. [63].

Previously, in liquid-liquid phase transfer reactions, the hydroxypropyl side-chain proved to be more effective than the methoxypropyl group in all cases [55]; this trend has persisted. Macrocycles with a methoxyphenylethyl substituent (1c and $\mathbf{2 c}$ ) also generated low enantiomeric excess values (19\% and 29\%, Table 1, entries 3 and 6). Comparing the data shows that the presence of a lipophilic side-chain negatively affects the catalytic activity in the Darzens condensation. Replacement of the benzylidene unit of catalysts $\mathbf{2 a - c}$ with ethyl groups resulted in similar catalytic activity. While using crown ether $\mathbf{2 a}$ gave the best ee value $(62 \%$, Table 1 , entry 4$)$, its $1 \mathbf{a}$ analogue generated somewhat lower enantioselectivity (52\%, Table 1, entry 1$)$ was the highest among the diethyl substituted macrocycles. It can be concluded that the rigidity is not the most crucial property of the carbohydrate-based crown ethers in the Darzens reaction.

Chiral epoxyketone 12 was also synthesized by epoxidation of trans-chalcone (13) under basic conditions (Scheme 4). Applying the crown catalysts, the highest enantioselectivity was again generated by $\mathbf{1} \mathbf{a}$ and $\mathbf{2 a}$ having a hydroxypropyl substituent $(75 \%$ ee and $92 \%$ ee, respectively; Table 2, entries 1 and 4). The same phenomenon was experienced as before, i.e., when a methoxypropyl group was attached to the nitrogen, ee values were low with macrocycles $\mathbf{1 b}$ and $\mathbf{2 b}$ ( $24 \%$ ee and $23 \%$ ee, Table 2, entries 2 and 5). Crown ether $\mathbf{2 c}$ bearing a methoxyphenylethyl group proved to be ineffective $(72 \mathrm{~h}, 3 \%$ ee, Table 2 , entry 6), while interestingly, its 1c analogue generated low but significantly higher enantiomeric excess in a shorter time ( $24 \mathrm{~h}, 21 \%$, Table 2 , entry 3$)$. In the case of $\mathbf{1 a}$ and $\mathbf{1 b}$, elongation of the reaction time was experienced ( $4 \mathrm{~h}$ for both, Table 2, entries 1 and 2) compared to catalysts $\mathbf{2 a}$ and $\mathbf{2 b}$ ( $1 \mathrm{~h}$ and $2 \mathrm{~h}$, respectively, Table 2, entries 4 and 5).

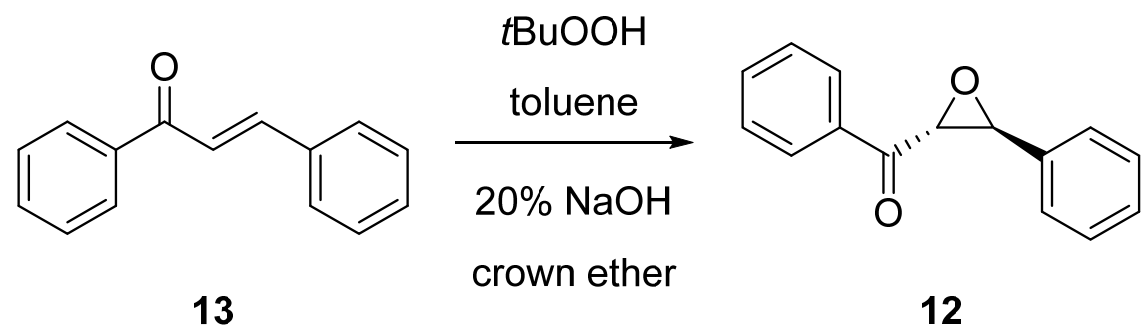

Scheme 4. Epoxidation of chalcone (13) with tert-butyl hydroperoxide in the presence of sugar-based crown ethers ( $\mathbf{1}$ or $\mathbf{2}$ ).

It can be concluded that the less rigid diethyl-substituted crown compounds (1a-c) showed lower efficiency in the epoxidation reaction than catalysts $\mathbf{2 a - c}$, having a benzylidene protecting group. However, comparing the results obtained with catalysts $1 \mathrm{c}$ and $2 c$, it can be seen that the effect of the side chain and that of the protecting group on the enantioselectivity are not independent of each other. 
Table 2. Effect of the crown catalysts (1 or 2 ) in the epoxidation of chalcone (13).

\begin{tabular}{ccccc}
\hline Entry & Catalyst & Time, $\mathbf{h}$ & Yield, $\mathbf{\%}^{\mathbf{a}}$ & $\mathbf{~ e e , ~} \mathbf{\%}^{\mathbf{b}}$ \\
\hline 1 & $\mathbf{1 a}$ & 4 & 79 & 75 \\
2 & $\mathbf{1 b}$ & 4 & 87 & 24 \\
3 & $\mathbf{1 c}$ & 24 & 70 & 21 \\
4 & $\mathbf{2 a}$ & 1 & 82 & $92^{\mathrm{c}}$ \\
5 & $\mathbf{2 b}$ & 2 & 61 & $23^{\mathrm{c}}$ \\
6 & $\mathbf{2 c}$ & 72 & 73 & 3 \\
\hline
\end{tabular}

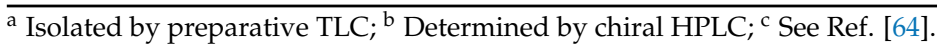

Asymmetric Michael addition offers an efficient method to prepare various products with new $\mathrm{C}-\mathrm{C}$ bonds using electron-deficient olefins and $\mathrm{CH}$-acidic compounds. The reaction of $\beta$-nitrostyrene (14) and diethyl acetamidomalonate (15) was investigated previously in our research group (Scheme 5). It has been found that using diethyl ether and THF in a ratio of $4: 1$ as the solvent significantly increases the enantiomeric excess generated by the sugar-based crown ether $\mathbf{2 a}$ (99\% ee, Table 3, entry 4) [66]. Its diethyl analogue 1a, however, showed only modest enantioselectivity under the same conditions ( $42 \%$ ee, Table 3 , entry 1). The absolute configuration of compound $\mathbf{1 6}$ was previously reported to be $S$ [71].

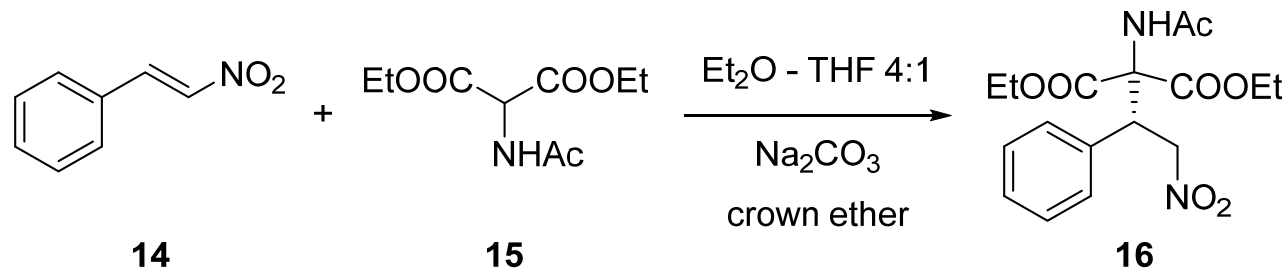

Scheme 5. Michael addition of diethyl acetamidomalonate (15) to $\beta$-nitrostyrene (14) the presence of sugar-based crown ethers ( $\mathbf{1}$ or $\mathbf{2})$.

Table 3. Effect of the glucose-based macrocycles ( 1 or 2 ) in Michael addition of $\beta$-nitrostyrene (14) and diethyl acetamidomalonate (15).

\begin{tabular}{ccccc}
\hline Entry & Catalyst & Time, $\mathbf{h}$ & Yield, $\mathbf{\%}^{\mathbf{a}}$ & $\mathbf{e e ,} \mathbf{\mathbf { o } ^ { \mathbf { b } }}$ \\
\hline 1 & $\mathbf{1 a}$ & 72 & 49 & 42 \\
2 & $\mathbf{1 b}$ & 48 & 42 & 28 \\
3 & $\mathbf{1 c}$ & 120 & 33 & 21 \\
4 & $\mathbf{2 a}$ & 3 & 70 & $99^{\mathrm{c}}$ \\
5 & $\mathbf{2 b}$ & 22 & 58 & 38 \\
6 & $\mathbf{2 c}$ & 120 & 36 & 15 \\
\hline
\end{tabular}

a Isolated by preparative TLC; ${ }^{\mathrm{b}}$ Determined by chiral HPLC; ${ }^{\mathrm{c}}$ See Ref. [65].

Asymmetric induction decreased again when a methoxypropyl sidearm was present (1b: $28 \%$ ee, $\mathbf{2 b}$ : $38 \%$ ee, Table 3, entries 2 and 5). Even lower ee values $(21 \%$ and $15 \%$, respectively, Table 3 , entries 3 and 6$)$ and significantly longer reaction times $(120 \mathrm{~h})$ were measured in the case of methoxyphenylethyl-substituted crown compounds $\mathbf{1} \mathbf{c}$ and $\mathbf{2 c}$.

Again, the replacement of the benzylidene moiety led to increased reaction times and lower asymmetric induction. The most striking difference was observed between lariat ether 1a and 2a when a highly enantioselective catalyst was converted into a less effective one with the change of the protecting group.

Finally, an enantioselective cyclopropanation reaction was investigated, in which two new $\mathrm{C}-\mathrm{C}$ bonds were formed in two steps. The first step is a Michael addition, followed by an intramolecular cyclization, while a leaving group is detached. Because of this mechanism, this reaction is called the Michael-initiated ring-closure (MIRC) reaction. In our model reaction, benzylidenemalononitrile (17) served as the Michael acceptor, and diethyl bromomalonate (18) was the $\mathrm{CH}$-acidic compound possessing a leaving group (Scheme 6). The absolute configuration of cyclopropane derivative 19 was previously assigned as $R$ [72]. 


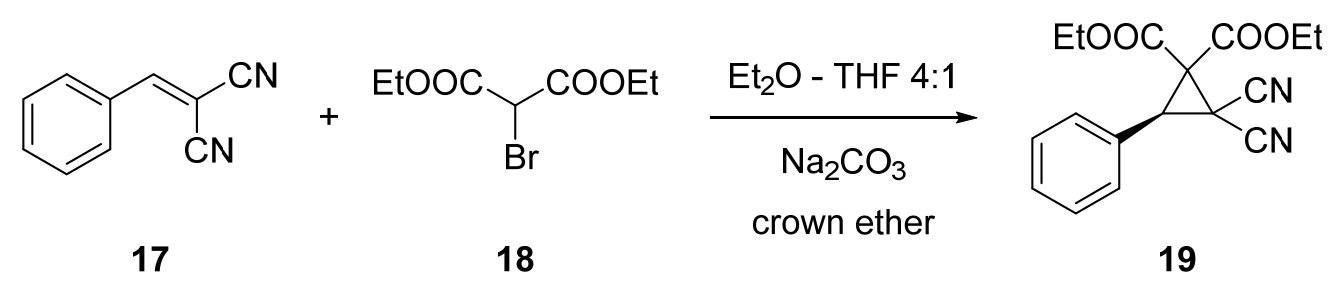

Scheme 6. Cyclopropanation reaction of benzylidenemalononitrile (17) with diethyl bromomalonate (18) in the presence of glucose-based catalysts ( 1 or 2 ).

As shown in Table 4, in this reaction, catalysts 1a and 2a showed only low enantioselective catalytic activity (22\% and 32\%, respectively, Table 4, entries 1 and 4 ). The presence of a methoxypropyl side-chain significantly increased the asymmetric induction. While in the case of crown ether $\mathbf{2} \mathbf{b}$, compound $\mathbf{1 9}$ was isolated with excellent yield $(97 \%)$ and good enantiomeric excess ( $70 \%$ ee) (Table 4 , entry 5$)$, the diethyl analogue $\mathbf{1 b}$ proved to be highly enantioselective in this reaction ( $99 \%$ ee, Table 4, entry 2$)$, however, the yield of cyclopropane derivative 19 was only moderate $(40 \%)$.

Table 4. Effect of the monoaza macrocycles (1 or 2 ) in the MIRC reaction of benzylidenemalononitrile (17) with diethyl bromomalonate (18).

\begin{tabular}{ccccc}
\hline Entry & Catalyst & Time, $\mathbf{h}$ & Yield, $\mathbf{\%}^{\mathbf{a}}$ & $\mathbf{~ e e , ~} \mathbf{\%}^{\mathbf{b}}$ \\
\hline 1 & $\mathbf{1 a}$ & 48 & 38 & 22 \\
2 & $\mathbf{1 b}$ & 24 & 40 & 99 \\
3 & $\mathbf{1 c}$ & 24 & 92 & 15 \\
4 & $\mathbf{2 a}$ & 20 & 82 & $32^{\mathrm{c}}$ \\
5 & $\mathbf{2 b}$ & 24 & 97 & 70 \\
6 & $\mathbf{2 c}$ & 24 & 81 & 58 \\
\hline
\end{tabular}

${ }^{\mathrm{a}}$ Isolated by preparative TLC; ${ }^{\mathrm{b}}$ Determined by chiral HPLC; ${ }^{\mathrm{c}}$ See Ref. [66].

There was a major difference between the effect of macrocycles 1c and 2c. Application of the former one (1c) led to a weak result ( $15 \%$ ee, Table 4 , entry 3$)$, while in the presence of $2 \mathbf{c}$ an ee of $58 \%$ was observed (Table 4 , entry 6 ). Again, these results strongly suggest that the side chain and the protecting group do not affect the enantioselectivity independently. With increased flexibility, catalysts 1c showed weaker result (15\% ee, Table 4, entry 3 ) than crown ether $2 \mathbf{c}(58 \%$ ee, Table 4 , entry 6$)$, while the less rigid lariat ether $\mathbf{1 b}$ was superior to macrocycle $\mathbf{2 b}$ (99\% ee and, 70\% ee, respectively, Table 4, entries 2 and 5).

\section{Materials and Methods}

\subsection{General}

Chemicals were purchased from Merck KGaA. Analytical and preparative thin-layer chromatography was performed on silica gel plates (60 GF-254, Merck, Kenilworth, NJ, USA), while column chromatography was carried out using 70-230 mesh silica gel and Brockman-II neutral aluminum oxide. Visualization of compounds on the TLC plates was performed using $254 \mathrm{~nm}$ UV light, iodine or $5 v / v \%$ sulfuric acid/methanol stain. Melting points were determined using a Stuart SMP10 apparatus and are uncorrected. The specific rotation was measured on a Perkin-Elmer $341 \mathrm{LC}$ polarimeter at $22{ }^{\circ} \mathrm{C}$ and $589 \mathrm{~nm}$. NMR spectra were obtained on a Bruker DRX-500 or Bruker-300 instrument in $\mathrm{CDCl}_{3}$ with $\mathrm{Me}_{4} \mathrm{Si}$ as an internal standard. HRMS measurements were performed using Q-TOF Premier mass spectrometer (Waters, Milford, MA, USA) in positive electrospray ionization mode. The enantiomeric excess values were determined on a PerkinElmer Series 200 liquid chromatography system using different columns. In all cases, isocratic elution was applied with a mobile phase flow rate of $0.8 \mathrm{~mL} / \mathrm{min}$. The temperature was $20^{\circ} \mathrm{C}$, and the wavelength of the detector was $254 \mathrm{~nm}$. 


\subsection{Synthesis of Crown Ethers}

3.2.1. Methyl-2,3-di-O-benzyl-4,6-di-O-ethyl- $\alpha$-D-glucopyranoside (6)

Methyl-2,3-di-O-benzyl- $\alpha$-D-glucopyranoside (5) (19.2 g, $51.3 \mathrm{mmol}$ ) was dissolved in dry tetrahydrofurane $(200 \mathrm{~mL})$ under argon atmosphere, and sodium hydride $(3.67 \mathrm{~g}$, $152.9 \mathrm{mmol}$ ) was added in small portions. The mixture was heated to reflux, and ethyl iodide $(31.8 \mathrm{~g}, 203.9 \mathrm{mmol})$ was added dropwise. TLC showed incomplete conversion after $30 \mathrm{~h}$ of reflux; thus, surplus reagents $(2.5 \mathrm{~g}, 104.2 \mathrm{mmol}$ sodium hydride; $31.8 \mathrm{~g}$, $203.9 \mathrm{mmol}$ ethyl iodide) were added and the mixture was refluxed for another $10 \mathrm{~h}$, after which conversion was complete. The reaction was quenched by dropwise addition of water $(10 \mathrm{~mL})$, and the mixture was concentrated in vacuum. The crude material was dissolved in a mixture of dichloromethane $(100 \mathrm{~mL})$, water $(40 \mathrm{~mL})$, and the phases were separated. The aqueous phase was extracted with dichloromethane $(2 \times 30 \mathrm{~mL})$, the extracts were combined with the organic phase, and this was washed with water (150 mL), dried $\left(\mathrm{Na}_{2} \mathrm{SO}_{4}\right)$, filtered, and concentrated in vacuum. The crude product was purified by column chromatography on a bed of silica gel $(350 \mathrm{~g})$ with hexane-ethyl acetate 3:2. Yield: $73 \%(16.05 \mathrm{~g})$, yellow, viscous oil. $[\alpha]_{D}^{25}=+71.6\left(c=1, \mathrm{CHCl}_{3}\right)$.

${ }^{1} \mathrm{H}$ NMR $\left(300 \mathrm{MHz}, \mathrm{CDCl}_{3}\right)$ \& 7.43-7.23 (m, 10H, $\left.\mathrm{ArH}\right) ; 5.00-4.78\left(\mathrm{~m}, 2 \mathrm{H}, \mathrm{ArCH}_{2} \mathrm{O}\right)$; 4.86-4.60 (m, 2H, ArCH $\left.\mathrm{A}_{2} \mathrm{O}\right) ; .61(\mathrm{~d}, J=3.6 \mathrm{~Hz}, 1 \mathrm{H}, \mathrm{H}-1)$; 3.96-3.78 (m, 2H, H-6a, H-5); 3.70-3.35 (m, 11H, H-2, H-4, H-3, H-6b, $\left.2 \times \mathrm{OCH}_{2}, \mathrm{OCH}_{3}\right) ; 1.22$ (t, $\left.J=7.0 \mathrm{~Hz}, 3 \mathrm{H}, \mathrm{CH}_{2} \mathrm{CH}_{3}\right)$; $1.19\left(\mathrm{t}, J=7.0 \mathrm{~Hz}, 3 \mathrm{H}, \mathrm{CH}_{2} \mathrm{CH}_{3}\right) ;{ }^{13} \mathrm{C} \mathrm{NMR}\left(300 \mathrm{MHz}^{\mathrm{CDCl}} \mathrm{CDCl}_{3}\right) \delta 139.99(\mathrm{ArC}) ; 138.29(\mathrm{ArC})$; 128.43 ( $\mathrm{ArC}) ; 128.37(\mathrm{ArC}) ; 127.98(\mathrm{ArC}) ; 127.87(\mathrm{ArC}) ; 127.56(\mathrm{ArC}) ; 98.29(\mathrm{C}-1) ; 82.07(\mathrm{CH})$; $79.67(\mathrm{CH}) ; 77.68(\mathrm{CH}) ; 75.72(\mathrm{CH}) ; 73.44\left(\mathrm{ArCH}_{2}\right) ; 70.12\left(\mathrm{ArCH}_{2}\right) ; 68.87\left(\mathrm{CH}_{2} \mathrm{CH}_{3}\right)$; $68.32\left(\mathrm{CH}_{2} \mathrm{CH}_{3}\right) ; 66.86\left(\mathrm{OCH}_{2} \mathrm{CH}\right) ; 55.12\left(\mathrm{OCH}_{3}\right) ; 15.83\left(\mathrm{CH}_{2} \mathrm{CH}_{3}\right) ; 15.11\left(\mathrm{CH}_{2} \mathrm{CH}_{3}\right)$.

HRMS calculated for $\mathrm{C}_{25} \mathrm{H}_{34} \mathrm{O}_{6} 430.2355$, found 430.2360 .

\subsubsection{Methyl-4,6-di-O-ethyl- $\alpha$-D-glucopyranoside (7)}

Methyl-2,3-di-O-benzyl-4,6-di-O-ethyl- $\alpha$-D-glucopyranoside (6) (16.05 g, $37.3 \mathrm{mmol}$ ) was subjected to hydrogenolysis in an autoclave in $100 \mathrm{~mL}$ of methanol using Selcat Q-6 type $\mathrm{Pd} / \mathrm{C}$ catalyst $(1.60 \mathrm{~g})$. After completion of the reaction, the mixture was filtered and concentrated. The crude product was dissolved in dichloromethane and filtered through fine filter paper to remove all traces of the catalyst. The resulting clear solution was concentrated in vacuum. Yield: $94 \%(8.78 \mathrm{~g})$, light-brown solid. $[\alpha]_{D}^{25}=+27.1\left(c=1, \mathrm{CHCl}_{3}\right)$. Mp. $79-83^{\circ} \mathrm{C}$.

${ }^{1} \mathrm{H}$ NMR $\left(300 \mathrm{MHz}, \mathrm{CDCl}_{3}\right) \delta 4.79(\mathrm{~d}, J=3.8 \mathrm{~Hz}, 1 \mathrm{H}, \mathrm{H}-1) ; 3.88-3.79(\mathrm{~m}, 1 \mathrm{H}, \mathrm{H}-$ 6a); $3.75(\mathrm{t}, J=9.3 \mathrm{~Hz}, 1 \mathrm{H}, \mathrm{H}-5) ; 3.71-3.46\left(\mathrm{~m}, 7 \mathrm{H}, \mathrm{H}-3, \mathrm{H}-4, \mathrm{H}-6 \mathrm{~b}, 2 \mathrm{x} \mathrm{OCH}_{2}\right) ; 3.41(\mathrm{~s}$, $\left.3 \mathrm{H}, \mathrm{OCH}_{3}\right) ; 3.34(\mathrm{t}, J=9.3 \mathrm{~Hz}, 1 \mathrm{H}, \mathrm{H}-2) ; 1.24\left(\mathrm{t}, J=7.0 \mathrm{~Hz}, 3 \mathrm{H}, \mathrm{CH}_{2} \mathrm{CH}_{3}\right) ; 1.21(\mathrm{t}$, $\left.J=7.0 \mathrm{~Hz}, 3 \mathrm{H}, \mathrm{CH}_{2} \mathrm{CH}_{3}\right) ;\left(\mathrm{OH}\right.$ groups were not visible); ${ }^{13} \mathrm{C} \mathrm{NMR}\left(300 \mathrm{MHz}, \mathrm{CDCl}_{3}\right) \delta$ 99.14 (C-1); 77.40 (CH); $75.10(\mathrm{CH}) ; 72.58(\mathrm{CH}) ; 70.28(\mathrm{CH}) ; 68.89\left(\mathrm{CH}_{2} \mathrm{CH}_{3}\right) ; 68.11\left(\mathrm{CH}_{2} \mathrm{CH}_{3}\right)$; $66.93\left(\mathrm{OCH}_{2} \mathrm{CH}\right) ; 55.29\left(\mathrm{OCH}_{3}\right) ; 15.76\left(\mathrm{CH}_{2} \mathrm{CH}_{3}\right) ; 15.11\left(\mathrm{CH}_{2} \mathrm{CH}_{3}\right)$.

HRMS calculated for $\mathrm{C}_{11} \mathrm{H}_{22} \mathrm{O}_{6} 250.1416$ found 250.1422 .

\subsubsection{Methyl-4,6-di-O-ethyl-2,3-bis-O-[(2-chloroethoxy)ethyl]- $\alpha$-D-glucopyranoside (8)}

A two-necked round-bottomed flask was fitted with a mechanical stirrer and was charged with methyl-4,6-di-O-ethyl- $\alpha$-D-glucopyranoside (7) $(8.78 \mathrm{~g}, 35.2 \mathrm{mmol})$ and bis(2-chloroethyl)ether (124 mL, $1.06 \mathrm{~mol}$ ). To this solution was added tetrabutylammonium hydrogensulfate ( $12.00 \mathrm{~g}, 35.2 \mathrm{mmol})$ and $50 \mathrm{~m} / \mathrm{m} \% \mathrm{NaOH}$ solution $(124 \mathrm{~mL} ; 94.6 \mathrm{~g}$, $2.36 \mathrm{~mol} \mathrm{NaOH})$. The resulting mixture was stirred vigorously for $12 \mathrm{~h}$, after which it was diluted with dichloromethane $(250 \mathrm{~mL})$ and water $(250 \mathrm{~mL})$. The phases were separated, the aqueous layer was extracted with dichloromethane $(4 \times 100 \mathrm{~mL})$. The combined organic phase was washed with water $(3 \times 100 \mathrm{~mL})$, dried over $\mathrm{Na}_{2} \mathrm{SO}_{4}$, and concentrated on a rotary evaporator. The excess bis(2-chloroethyl)ether was removed by vacuum distillation, and the crude product $(18.33 \mathrm{~g}$ ) was purified by column chromatography on a bed of silica gel (370 g). Gradient elution was used, $\mathrm{CHCl}_{3} \rightarrow \mathrm{CHCl}_{3}-\mathrm{MeOH}$ 100:2. Yield: $72 \%$ (11.74 g), orange, viscous oil. $[\alpha]_{D}^{25}=+65.1\left(c=1, \mathrm{CHCl}_{3}\right)$. 
${ }^{1} \mathrm{H}$ NMR $\left(300 \mathrm{MHz} \mathrm{CDCl}_{3}\right) \delta 4.83(\mathrm{~d}, J=3.6 \mathrm{~Hz}, 1 \mathrm{H}, \mathrm{H}-1) ; 4.04-3.96$ (m, 1H, H-6a); 3.94-3.72 (m, 9H, H-3, H-5, H-6b, $\left.3 \times \mathrm{OCH}_{2}\right)$; 3.69-3.56 (m, 13H, H-4, $4 \times \mathrm{OCH}_{2}, 2$ x CH $3.49(\mathrm{dd}, J=9.4 ; 7.0 \mathrm{~Hz}, 1 \mathrm{H}, \mathrm{H}-2) ; 3.43-3.34\left(\mathrm{~m}, 5 \mathrm{H}, \mathrm{OCH}_{2}, \mathrm{OCH}_{3}\right) ; 1.22(\mathrm{t}, J=7.0 \mathrm{~Hz}, 3 \mathrm{H}$, $\left.\mathrm{CH}_{2} \mathrm{CH}_{3}\right) ; 1.20\left(\mathrm{t}, \mathrm{J}=7.0 \mathrm{~Hz}, 3 \mathrm{H}, \mathrm{CH}_{2} \mathrm{CH}_{3}\right) ;{ }^{13} \mathrm{C} \mathrm{NMR}\left(300 \mathrm{MHz}, \mathrm{CDCl}_{3}\right) \delta 92.23(\mathrm{C}-1)$; $82.61(\mathrm{CH}) ; 81.09(\mathrm{CH}) ; 77.70(\mathrm{CH}) ; 72.54(\mathrm{CH}) ; 71.50\left(\mathrm{OCH}_{2} \mathrm{CH}_{2}\right) ; 71.41\left(\mathrm{OCH}_{2} \mathrm{CH}_{2}\right) ; 71.18$ $\left(\mathrm{OCH}_{2} \mathrm{CH}_{2}\right) ; 71.08\left(\mathrm{OCH}_{2} \mathrm{CH}_{2}\right) ; 70.98\left(\mathrm{OCH}_{2} \mathrm{CH}_{2}\right) ; 70.32\left(\mathrm{OCH}_{2} \mathrm{CH}_{2}\right) ; 69.10\left(\mathrm{CH}_{2} \mathrm{CH}_{3}\right)$; $68.47\left(\mathrm{CH}_{2} \mathrm{CH}_{3}\right) ; 67.06\left(\mathrm{OCH}_{2} \mathrm{CH}\right) ; 55.21\left(\mathrm{OCH}_{3}\right) ; 42.95\left(\mathrm{CH}_{2} \mathrm{CH}_{2} \mathrm{Cl}\right) ; 42.90\left(\mathrm{CH}_{2} \mathrm{CH}_{2} \mathrm{Cl}\right)$; $16.02\left(\mathrm{CH}_{2} \mathrm{CH}_{3}\right) ; 15.31\left(\mathrm{CH}_{2} \mathrm{CH}_{3}\right)$.

HRMS calculated for $\mathrm{C}_{19} \mathrm{H}_{36} \mathrm{Cl}_{2} \mathrm{O}_{8} 462.1718$, found 462.1722 .

\subsubsection{Methyl-4,6-di-O-ethyl-2,3-bis-O-[(2-iodoethoxy)ethyl]- $\alpha$-D-glucopyranoside (9)}

Methyl-4,6-di-O-ethyl-2,3-bis-O-[(2-chloroethoxy)ethyl]- $\alpha$-D-glucopyranoside (8) (11.70 g, $25.3 \mathrm{mmol})$ was dissolved in dry acetone $(150 \mathrm{~mL})$. Sodium iodide $(15.00 \mathrm{~g}, 100.1 \mathrm{mmol})$ was added, and the mixture was refluxed for $50 \mathrm{~h}$, during which a white precipitate was formed. The reaction mixture was filtered and concentrated. The crude product was dissolved in dichloromethane and washed with water $(4 \times 40 \mathrm{~mL})$, dried over $\mathrm{Na}_{2} \mathrm{SO}_{4}$, and concentrated again. Yield: $84 \%$ (13.77 g), brown, viscous oil. $[\alpha]_{D}^{25}=+49.5\left(c=1, \mathrm{CHCl}_{3}\right)$.

${ }^{1} \mathrm{H}$ NMR (300 MHz, $\left.\mathrm{CDCl}_{3}\right) \delta 4.82(\mathrm{~d}, J=3.5 \mathrm{~Hz}, 1 \mathrm{H}, \mathrm{H}-1) ; 4.02-3.95$ (m, 1H, H-6a); 3.92-3.82 (m, 3H, H-5, OCH $)$; 3.92-3.70 (m, 6H, H-3, H-6b, 2 x OCH$)$; 3.69-3.54 (m, 9H, $\left.\mathrm{H}-4,4 \times \mathrm{OCH}_{2}\right)$; 3.53-3.43 (m, 1H, H-2); 3.43-3.33 (m, 5H, OCH $\left.\mathrm{OCH}_{3}\right) ; 3.30-3.20(\mathrm{~m}$, $\left.4 \mathrm{H}, 2 \times \mathrm{CH}_{2} \mathrm{I}\right) ; 1.1\left(\mathrm{t}, J=7.0 \mathrm{~Hz}, 3 \mathrm{H}, \mathrm{CH}_{2} \mathrm{CH}_{3}\right) ; 1.19\left(\mathrm{t}, J=7.0 \mathrm{~Hz}, 3 \mathrm{H}, \mathrm{CH}_{2} \mathrm{CH}_{3}\right) ;{ }^{13} \mathrm{C}$ NMR (300 MHz, $\left.\mathrm{CDCl}_{3}\right) \delta 98.25(\mathrm{C}-1) ; 82.63(\mathrm{CH}) ; 81.04(\mathrm{CH}) ; 77.70(\mathrm{CH}) ; 72.57(\mathrm{CH})$; $72.12\left(\mathrm{OCH}_{2} \mathrm{CH}_{2}\right) ; 72.07\left(\mathrm{OCH}_{2} \mathrm{CH}_{2}\right) ; 71.12\left(\mathrm{OCH}_{2} \mathrm{CH}_{2}\right) ; 70.78\left(\mathrm{OCH}_{2} \mathrm{CH}_{2}\right) ; 70.59\left(\mathrm{OCH}_{2} \mathrm{CH}_{2}\right)$; $70.33\left(\mathrm{OCH}_{2} \mathrm{CH}_{2}\right) ; 69.09\left(\mathrm{CH}_{2} \mathrm{CH}_{3}\right) ; 68.50\left(\mathrm{CH}_{2} \mathrm{CH}_{3}\right) ; 67.08\left(\mathrm{OCH}_{2} \mathrm{CH}\right) ; 55.25\left(\mathrm{OCH}_{3}\right)$; $16.08\left(\mathrm{CH}_{2} \mathrm{CH}_{3}\right) ; 15.33\left(\mathrm{CH}_{2} \mathrm{CH}_{3}\right) ; 3.10\left(\mathrm{CH}_{2} \mathrm{CH}_{2} \mathrm{I}\right) ; 3.01\left(\mathrm{CH}_{2} \mathrm{CH}_{2} \mathrm{I}\right)$.

HRMS calculated for $\mathrm{C}_{19} \mathrm{H}_{36} \mathrm{I}_{2} \mathrm{O}_{8}$ 646.0500, found 646.0507.

3.2.5. Methyl-4,6-di-O-ethyl-2,3-dideoxy- $\alpha$-D-glucopyranosido[2,3-h]-N-[3hydroxypropyl]-1,4,7,10-tetraoxa-13-azacyclopentadecane (1a)

Methyl-4,6-di-O-ethyl-2,3-bis-O-[(2-iodoethoxy)ethyl]- $\alpha$-D-glucopyranoside (9) (2.80 g, $4.33 \mathrm{mmol})$ was dissolved in dry acetonitrile $(60 \mathrm{~mL})$, then 3-aminopropanol $(0.33 \mathrm{~g}$, $4.33 \mathrm{mmol})$ and $\mathrm{Na}_{2} \mathrm{CO}_{3}(2.76 \mathrm{~g}, 26.0 \mathrm{mmol})$ were added. The mixture was refluxed under Ar atmosphere for $40 \mathrm{~h}$. Upon completion of the reaction, the mixture was filtered, and the filtrate was concentrated. The crude product was dissolved in dichloromethane and washed with water $(3 \times 20 \mathrm{~mL})$, then the aqueous phase was extracted with dichloromethane $(2 \times 20 \mathrm{~mL})$, and the organic phases were combined. This combined organic phase was dried over $\mathrm{Na}_{2} \mathrm{SO}_{4}$ and concentrated in vacuum affording $1.75 \mathrm{~g}$ of crude product. This was purified by column chromatography on an aluminum-oxide bed (52.5 g). Gradient elution was used $\mathrm{CH}_{2} \mathrm{Cl}_{2} \rightarrow \mathrm{CH}_{2} \mathrm{Cl}_{2}-\mathrm{MeOH}$ 100:1.

Yield: $65 \%(1.30 \mathrm{~g})$, yellow, viscous oil. $[\alpha]_{D}^{25}=+68.3\left(c=1, \mathrm{CHCl}_{3}\right)$.

${ }^{1} \mathrm{H}$ NMR $\left(300 \mathrm{MHz}, \mathrm{CDCl}_{3}\right) \delta 4.83(\mathrm{~d}, J=3.6 \mathrm{~Hz}, 1 \mathrm{H}, \mathrm{H}-1) ; 4.15-4.06(\mathrm{~m}, 1 \mathrm{H}, \mathrm{H}-$ 6a); 3.85-3.53 (m, 20H, H-3, H-4, H-5, H-6b, 7 x OCH, $\left.\mathrm{CH}_{2} \mathrm{OH}\right) ; 3.53-3.44$ (m, 1H, H2); 3.43-3.33 (m, 5H, $\left.\mathrm{OCH}_{3}, \mathrm{OCH}_{2}\right) ; 3.00-2.64\left(\mathrm{~m}, 6 \mathrm{H}, 3 \times \mathrm{NCH}_{2}\right) ; 1.75-1.67(\mathrm{~m}, 2 \mathrm{H}$, $\left.\mathrm{CH}_{2} \mathrm{CH}_{2} \mathrm{CH}_{2}\right) ; 1.22\left(\mathrm{t}, \mathrm{J}=7.0 \mathrm{~Hz}, 3 \mathrm{H}, \mathrm{CH}_{2} \mathrm{CH}_{3}\right) ; 1.18\left(\mathrm{t}, \mathrm{J}=7.0 \mathrm{~Hz}, 3 \mathrm{H}, \mathrm{CH}_{2} \mathrm{CH}_{3}\right) ;{ }^{13} \mathrm{C}$ NMR (300 MHz, $\left.\mathrm{CDCl}_{3}\right) \delta 97.58(\mathrm{C}-1) ; 81.85(\mathrm{CH}) ; 80.28(\mathrm{CH}) ; 77.98(\mathrm{CH}) ; 72.79(\mathrm{CH})$; $70.81\left(2 \times \mathrm{OCH}_{2} \mathrm{CH}_{2}\right) ; 70.38\left(2 \times \mathrm{OCH}_{2} \mathrm{CH}_{2}\right) ; 70.28\left(2 \times \mathrm{OCH}_{2} \mathrm{CH}_{2}\right) ; 69.12\left(\mathrm{CH}_{2} \mathrm{CH}_{3}\right) ; 68.44$ $\left(\mathrm{CH}_{2} \mathrm{CH}_{3}\right) ; 67.11\left(\mathrm{OCH}_{2} \mathrm{CH}\right) ; 59.77\left(\mathrm{CH}_{2} \mathrm{OH}\right) ; 55.20\left(\mathrm{OCH}_{3}\right) ; 54.70\left(2 \mathrm{xNCH}_{2}\right) ; 54.56\left(\mathrm{NCH}_{2}\right)$; $29.94\left(\mathrm{NCH}_{2} \mathrm{CH}_{2} \mathrm{CH}_{2} \mathrm{OH}\right) ; 16.08\left(\mathrm{CH}_{2} \mathrm{CH}_{3}\right) ; 15.36\left(\mathrm{CH}_{2} \mathrm{CH}_{3}\right)$.

HRMS calculated for $\mathrm{C}_{22} \mathrm{H}_{43} \mathrm{NO}_{9} 465.2938$, found 465.2940.

3.2.6. Methyl-4,6-di-O-ethyl-2,3-dideoxy- $\alpha$-D-glucopyranosido[2,3-h]-N-[3methoxypropyl]-1,4,7,10-tetraoxa-13-azacyclopentadecane (1b)

Methyl-4,6-di-O-ethyl-2,3-bis-O-[(2-iodoethoxy)ethyl]- $\alpha$-D-glucopyranoside (9) (2.80 g, $4.33 \mathrm{mmol})$ was dissolved in dry acetonitrile $(60 \mathrm{~mL})$, then 3-methoxypropylamine $(0.39 \mathrm{~g}$, 
$4.33 \mathrm{mmol})$ and $\mathrm{Na}_{2} \mathrm{CO}_{3}(2.76 \mathrm{~g}, 26.0 \mathrm{mmol})$ were added. The mixture was refluxed under Ar atmosphere for $40 \mathrm{~h}$. Upon completion of the reaction, the mixture was filtered, and the filtrate was concentrated. The crude product was dissolved in dichloromethane and washed with water $(2 \times 25 \mathrm{~mL})$, then the water washings were extracted with dichloromethane $(20 \mathrm{~mL})$, and the organic phases were combined. This combined organic phase was dried over $\mathrm{Na}_{2} \mathrm{SO}_{4}$ and concentrated in vacuum affording $2.16 \mathrm{~g}$ of crude product. This was purified by column chromatography on a silica gel bed ( $45 \mathrm{~g})$. Gradient elution was used $\mathrm{CH}_{2} \mathrm{Cl}_{2} \rightarrow \mathrm{CH}_{2} \mathrm{Cl}_{2}-\mathrm{MeOH}$ 100:8.

Yield: $69 \%(1.43 \mathrm{~g})$, brown, viscous oil. $[\alpha]_{D}^{25}=+63.1\left(c=1, \mathrm{CHCl}_{3}\right)$.

${ }^{1} \mathrm{H}$ NMR $\left(300 \mathrm{MHz}, \mathrm{CDCl}_{3}\right) \delta 4.82(\mathrm{~d}, J=3.5 \mathrm{~Hz}, 1 \mathrm{H}, \mathrm{H}-1) ; 4.16-4.08(\mathrm{~m}, 1 \mathrm{H}, \mathrm{H}-6 \mathrm{a})$; 3.85-3.51 (m, 18H, H-3, H-4, H-5, H-6b, 7 x OCH 2 ); 3.50-3.43 (m, 1H, H-2); 3.43-3.33 (m, $\left.7 \mathrm{H}, 2 \times \mathrm{OCH}_{2}, \mathrm{OCH}_{3}\right) ; 3.30\left(\mathrm{~s}, 3 \mathrm{H}, \mathrm{CH}_{2} \mathrm{OCH}_{3}\right) ; 2.89-2.50\left(\mathrm{~m}, 6 \mathrm{H}, 3 \times \mathrm{NCH}_{2}\right) ; 1.78-1.70(\mathrm{~m}$, $\left.2 \mathrm{H}, \mathrm{CH}_{2} \mathrm{CH}_{2} \mathrm{CH}_{2}\right) ; 1.20\left(\mathrm{t}, J=7.0 \mathrm{~Hz}, 3 \mathrm{H}, \mathrm{CH}_{2} \mathrm{CH}_{3}\right) ; 1.16\left(\mathrm{t}, J=7.0 \mathrm{~Hz}, 3 \mathrm{H}, \mathrm{CH}_{2} \mathrm{CH}_{3}\right)$; ${ }^{13} \mathrm{C}$ NMR (300 MHz. CDCl 3 ) $\delta 97.52(\mathrm{C}-1) ; 81.82(\mathrm{CH}) ; 80.38(\mathrm{CH}) ; 77.85(\mathrm{CH}) ; 72.68(\mathrm{CH})$; $70.82\left(2 \times \mathrm{OCH}_{2} \mathrm{CH}_{2}\right) ; 70.33\left(2 \times \mathrm{OCH}_{2} \mathrm{CH}_{2}\right) ; 70.29\left(2 \times \mathrm{OCH}_{2} \mathrm{CH}_{2}\right) ; 69.07\left(\mathrm{CH}_{2} \mathrm{CH}_{3}\right)$; $68.43\left(\mathrm{CH}_{2} \mathrm{CH}_{3}\right) ; 67.07\left(\mathrm{OCH}_{2} \mathrm{CH}\right) ; 58.81\left(\mathrm{CH}_{2} \mathrm{OCH}_{3}\right) ; 55.17\left(2 \times \mathrm{OCH}_{3}\right) ; 53.74\left(3 \mathrm{x} \mathrm{NCH}_{2}\right)$; $29.90\left(\mathrm{NCH}_{2} \mathrm{CH}_{2} \mathrm{CH}_{2} \mathrm{OCH}_{3}\right) ; 16.06\left(\mathrm{CH}_{2} \mathrm{CH}_{3}\right) ; 15.33\left(\mathrm{CH}_{2} \mathrm{CH}_{3}\right)$.

HRMS calculated for $\mathrm{C}_{23} \mathrm{H}_{45} \mathrm{NO}_{9} 479.3094$, found 479.3095 .

3.2.7. Methyl-4,6-di-O-ethyl-2,3-dideoxy- $\alpha$-D-glucopyranosido[2,3-h]- $N-[2-(2-$ methoxyphenyl)ethyl]-1,4,7,10-tetraoxa-13-azacyclopentadecane (1c)

Methyl-4,6-di-O-ethyl-2,3-bis-O-[(2-iodoethoxy)ethyl]- $\alpha$-D-glucopyranoside (9) (2.80 g, $4.33 \mathrm{mmol})$ was dissolved in dry acetonitrile $(60 \mathrm{~mL})$, then 2-(2-methoxyphenyl)ethylamine $(0.66 \mathrm{~g}, 4.33 \mathrm{mmol})$ and $\mathrm{Na}_{2} \mathrm{CO}_{3}(2.76 \mathrm{~g}, 26.0 \mathrm{mmol})$ were added. The mixture was refluxed under Ar atmosphere for $40 \mathrm{~h}$. Upon completion of the reaction, the mixture was filtered, and the filtrate was concentrated. The crude product was dissolved in dichloromethane and washed with water $(2 \times 25 \mathrm{~mL})$, then the water washings were extracted with dichloromethane $(20 \mathrm{~mL})$, and the organic phases were combined. This combined organic phase was dried over $\mathrm{Na}_{2} \mathrm{SO}_{4}$ and concentrated in vacuum affording $2.59 \mathrm{~g}$ of crude product. This was purified by column chromatography on a silica gel bed (77 g). Gradient elution was used $\mathrm{CH}_{2} \mathrm{Cl}_{2} \rightarrow \mathrm{CH}_{2} \mathrm{Cl}_{2}-\mathrm{MeOH}$ 100:4.

Yield: $60 \%(1.39 \mathrm{~g})$, brown, viscous oil. $[\alpha]_{D}^{25}=+54.8\left(c=1, \mathrm{CHCl}_{3}\right)$.

${ }^{1} \mathrm{H}$ NMR $\left(300 \mathrm{MHz}, \mathrm{CDCl}_{3}\right) \delta 7.22-7.11(\mathrm{~m}, 2 \mathrm{H}, \mathrm{ArH}) ; 6.90-6.80(\mathrm{~m}, 2 \mathrm{H}, \mathrm{ArH}) ; 4.83(\mathrm{~d}$, $J=3.4 \mathrm{~Hz}, 1 \mathrm{H}, \mathrm{H}-1) ; 4.12(\mathrm{t}, J=9.4 \mathrm{~Hz}, 1 \mathrm{H}, \mathrm{H}-6 \mathrm{a}) ; 3.85-3.52(\mathrm{~m}, 21 \mathrm{H}, \mathrm{H}-3, \mathrm{H}-4, \mathrm{H}-5, \mathrm{H}-6 \mathrm{~b}$, $\left.\mathrm{ArOCH}_{3}, 7 \times \mathrm{OCH}_{2}\right) ; 3.52-3.43(\mathrm{~m}, 1 \mathrm{H}, \mathrm{H}-2) ; 3.43-3.33\left(\mathrm{~m}, 5 \mathrm{H}, \mathrm{OCH}_{2}, \mathrm{OCH}_{3}\right) ; 2.92-2.51(\mathrm{~m}$, $\left.8 \mathrm{H}, 3 \times \mathrm{NCH}_{2}, \mathrm{ArCH}_{2}\right) ; 1.20\left(\mathrm{t}, J=7.0 \mathrm{~Hz}, 3 \mathrm{H}, \mathrm{CH}_{2} \mathrm{CH}_{3}\right) ; 1.17\left(\mathrm{t}, J=7.0 \mathrm{~Hz}, 3 \mathrm{H}, \mathrm{CH}_{2} \mathrm{CH}_{3}\right)$; ${ }^{13} \mathrm{C} \mathrm{NMR}\left(300 \mathrm{MHz} . \mathrm{CDCl}_{3}\right) \delta 157.41\left(\mathrm{ArCOCH}_{3}\right) ; 130.54(\mathrm{ArC}) ; 129.66(\mathrm{ArC}) ; 123.94(\mathrm{ArC}) ;$ 120.62 (ArC); $110.28(\mathrm{ArC}) ; 97.27(\mathrm{C}-1) ; 81.55(\mathrm{CH}) ; 80.08(\mathrm{CH}) ; 77.74(\mathrm{CH}) ; 72.49(\mathrm{CH})$; $70.83\left(2 \times \mathrm{OCH}_{2} \mathrm{CH}_{2}\right) ; 70.17\left(2 \times \mathrm{OCH}_{2} \mathrm{CH}_{2}\right) ; 70.15\left(2 \times \mathrm{OCH}_{2} \mathrm{CH}_{2}\right) ; 70.03\left(\mathrm{CH}_{2} \mathrm{CH}_{3}\right)$; $68.85\left(\mathrm{CH}_{2} \mathrm{CH}_{3}\right) ; 68.23\left(\mathrm{OCH}_{2} \mathrm{CH}\right) ; 66.87\left(\mathrm{NCH}_{2}\right) ; 55.30\left(2 \mathrm{x} \mathrm{OCH}_{3}\right) ; 54.95\left(2 \mathrm{xCH}_{2}\right)$; $29.70\left(\mathrm{NCH}_{2} \mathrm{CH}_{2} \mathrm{CH}_{2} \mathrm{OPhOCH}_{3}\right) ; 15.86\left(\mathrm{CH}_{2} \mathrm{CH}_{3}\right) ; 15.13\left(\mathrm{CH}_{2} \mathrm{CH}_{3}\right)$.

HRMS calculated for $\mathrm{C}_{28} \mathrm{H}_{47} \mathrm{NO}_{9} 541.3251$ found 541.3255.

\subsection{Asymmetric Reactions}

3.3.1. Synthesis of (2R,3S)-Phenyl(3-phenyloxirane-2-yl)methanone (12) via Darzens Condensation

2-Chloroacetophenone $(0.15 \mathrm{~g}, 1 \mathrm{mmol})$ and benzaldehyde $(0.15 \mathrm{~mL}, 1.5 \mathrm{mmol})$ and the appropriate crown catalyst $(10 \mathrm{~mol} \%)$ were dissolved in toluene $(3 \mathrm{~mL})$. Then $30 \%$ aq. $\mathrm{NaOH}$ solution $(1 \mathrm{~mL})$ was added, and the mixture was stirred at room temperature. The reaction was monitored by TLC (hexane-ethyl-acetate 10:1). After completing the reaction, the mixture was diluted with toluene $(7 \mathrm{~mL})$ and water $(3 \mathrm{~mL})$, and the phases were separated. The organic layer was washed with $10 \%$ aq. $\mathrm{HCl}$ solution $(3 \times 10 \mathrm{~mL})$, dried $\left(\mathrm{Na}_{2} \mathrm{CO}_{3}\right.$ and $\left.\mathrm{Na}_{2} \mathrm{SO}_{4}\right)$, filtered, and concentrated in vacuum. The crude product was purified by preparative TLC (hexane-ethyl-acetate 10:1) to give a yellowish-white 
powder with an mp of $64-66^{\circ} \mathrm{C}$. For the respective yields and ee values, see Table 1 . Chiral HPLC: Phenomenex Lux ${ }^{\circledR} 5 \mathrm{u}$ Cellulose-1 column, hexane:EtOH 85:15, major enantiomer $\mathrm{t}_{\mathrm{R}}=9.5 \mathrm{~min}$, minor enantiomer $\mathrm{t}_{\mathrm{R}}=8.2 \mathrm{~min} .[\alpha]_{D}^{22}=-132.7\left(c=1, \mathrm{CH}_{2} \mathrm{Cl}_{2}\right)(62 \%$ ee $)$

${ }^{1} \mathrm{H}$ NMR $\left(\mathrm{CDCl}_{3}, 500 \mathrm{MHz}\right), \delta[\mathrm{ppm}]:$ 7.97-7.94 (m, 2H, ArH), 7.60-7.56 (m, 1H, ArH), 7.46-7.44 (m, 2H, ArH), 7.38-7.32 (m, 5H), $4.26(\mathrm{~d}, J=1.9 \mathrm{~Hz}, 1 \mathrm{H}, \mathrm{COCH}), 4.05(\mathrm{~d}, J=1.9 \mathrm{~Hz}$, $1 \mathrm{H}, \mathrm{ArCH}) ;{ }^{13} \mathrm{C}$ NMR $\left(75 \mathrm{MHz} \mathrm{CDCl}_{3}\right), \delta[\mathrm{ppm}]: 193.06(\mathrm{C}=\mathrm{O}), 135.48(\mathrm{ArC}), 133.97(\mathrm{ArC})$, $129.04(\mathrm{ArC}), 128.86$ (ArC), $128.76(\mathrm{ArC}), 128.33(\mathrm{ArC}), 125.78$ (ArC), 61.00 (OCCO), $59.34(\mathrm{PhCO})$.

HRMS calculated for $\mathrm{C}_{15} \mathrm{H}_{12} \mathrm{O}_{2} 224.0837$, found 224.0840 .

3.3.2. Synthesis of (2R,3S)-Phenyl(3-phenyloxirane-2-yl)methanone (12) via Epoxidation of trans-Chalcone

trans-Chalcone $(0.25 \mathrm{~g}, 1.2 \mathrm{mmol})$ and the appropriate crown catalyst $(10 \mathrm{~mol} \%)$ were dissolved in toluene $(3 \mathrm{~mL})$, then $5.5 \mathrm{M}$ tert-butylhydroperoxide solution $(0.5 \mathrm{~mL}$, in decane) and $20 \%$ aq. $\mathrm{NaOH}$ solution $(1 \mathrm{~mL})$ was added. The mixture was stirred at room temperature. The reaction was monitored by TLC (hexane-ethyl-acetate 10:1). After completion, the reaction mixture was diluted with toluene $(7 \mathrm{~mL})$ and water $(3 \mathrm{~mL})$, and the phases were separated. The organic layer was washed with $10 \%$ aq. $\mathrm{HCl}$ solution $(3 \times 10 \mathrm{~mL})$, dried $\left(\mathrm{Na}_{2} \mathrm{CO}_{3}\right.$ and $\left.\mathrm{Na}_{2} \mathrm{SO}_{4}\right)$, filtered, and concentrated in vacuum. The crude product was purified by preparative TLC (hexane-ethyl-acetate 10:1) to give a yellowish-white powder having an $\mathrm{mp}$ of $64-66^{\circ} \mathrm{C}$. For the respective yields and ee values, see Table 2. Chiral HPLC: Phenomenex Lux ${ }^{\circledR} 5$ u Cellulose-1 column, hexane:EtOH 85:15, major enantiomer $t_{R}=8.2 \mathrm{~min}$, minor enantiomer $t_{R}=9.4 \mathrm{~min} . \quad[\alpha]_{D}^{22}=-196.8(c=1$, $\left.\mathrm{CH}_{2} \mathrm{Cl}_{2}\right)(92 \%$ ee$)$.

${ }^{1} \mathrm{H}$ NMR $\left(\mathrm{CDCl}_{3}, 500 \mathrm{MHz}\right), \delta$ [ppm]: 7.97-7.94 (m, 2H, ArH), 7.60-7.56 (m, 1H, ArH), 7.46-7.44 (m, 2H, ArH), 7.38-7.32 (m, 5H), $4.26(\mathrm{~d}, J=1.9 \mathrm{~Hz}, 1 \mathrm{H}, \mathrm{COCH}), 4.05(\mathrm{~d}, J=1.9 \mathrm{~Hz}$, $1 \mathrm{H}, \mathrm{ArCH}) ;{ }^{13} \mathrm{C} \mathrm{NMR}\left(75 \mathrm{MHz}, \mathrm{CDCl}_{3}\right), \delta$ [ppm]: $193.06(\mathrm{C}=\mathrm{O}), 135.48(\mathrm{ArC}), 133.97(\mathrm{ArC})$, $129.04(\mathrm{ArC}), 128.86(\mathrm{ArC}), 128.76(\mathrm{ArC}), 128.33(\mathrm{ArC}), 125.78$ (ArC), $61.00(\mathrm{OCCO})$, 59.34 (PhCO).

HRMS calculated for $\mathrm{C}_{15} \mathrm{H}_{12} \mathrm{O}_{2} 224.0837$, found 224.0839 .

3.3.3. Synthesis of (S)-Diethyl 2-Acetamido-2-(2-nitro-1-phenylethyl)malonate (16) via Michael Addition

Diethyl acetamidomalonate $(1.5 \mathrm{mmol}), \beta$-nitrostyrene $(1.0 \mathrm{mmol})$, and the appropriate crown catalyst $(10 \mathrm{~mol} \%)$ were dissolved in a 4:1 mixture of dry diethyl ether and dry THF. After a short period of stirring, anhydrous $\mathrm{Na}_{2} \mathrm{CO}_{3}(0.20 \mathrm{~g}, 1.9 \mathrm{mmol})$ was added, and the mixture was stirred at room temperature. The reaction was monitored by TLC (hexane-ethyl-acetate 5:1). After completion, the solvents were removed in vacuum; the residue was dissolved in dichloromethane and filtered. The filtrate was washed with $10 \%$ aq $\mathrm{HCl}(3 \times 10 \mathrm{~mL})$ and dried $\left(\mathrm{Na}_{2} \mathrm{CO}_{3}\right.$ and $\left.\mathrm{Na}_{2} \mathrm{SO}_{4}\right)$. The crude product obtained after evaporating the solvent was purified by preparative TLC (hexane-ethyl-acetate 10:1) to give an off-white solid having an $\mathrm{mp}$ of $135-136^{\circ} \mathrm{C}$. For the respective yields and ee values, see Table 3. Chiral HPLC: Phenomenex Lux ${ }^{\circledR} 5 \mathrm{u}$ Amylose-2 column, hexane:EtOH 85:15, major enantiomer $t_{R}=16.8 \mathrm{~min}$, minor enantiomer $t_{\mathrm{R}}=18.6 \mathrm{~min} .=-42.8\left(\mathrm{c}=1, \mathrm{CHCl}_{3}\right)$ (99\% ee).

${ }^{1} \mathrm{H}$ NMR (500 MHz, $\left.\mathrm{CDCl}_{3}\right) \delta(\mathrm{ppm}): 7.31-7.28(\mathrm{~m}, 3 \mathrm{H}, \mathrm{ArH}), 7.22-7.18(\mathrm{~m}, 2 \mathrm{H}, \mathrm{ArH})$, 6.89 (br s, NH), 5.54-5.48 (m, 1H, PhCH), 4.73-4.66 (m, 2H, OCH $)_{2}, 4.34-4.23\left(\mathrm{~m}, 2 \mathrm{H}, \mathrm{OCH}_{2}\right)$, 4.20-4.13 (m, 1H, CH $\left.\mathrm{NO}_{2}\right), 4.08-4.01\left(\mathrm{~m}, 1 \mathrm{H}, \mathrm{CH}_{2} \mathrm{NO}_{2}\right), 2.12\left(\mathrm{~s}, 3 \mathrm{H}, \mathrm{COCH}_{3}\right), 1.27(\mathrm{t}$, $\left.J=7 \mathrm{~Hz}, 3 \mathrm{H}, \mathrm{CH}_{3} \mathrm{CH}_{2}\right), 1.25\left(\mathrm{t}, J=7 \mathrm{~Hz}, 3 \mathrm{H}, \mathrm{CH}_{3} \mathrm{CH}_{2}\right) ;{ }^{13} \mathrm{C} \mathrm{NMR}\left(75 \mathrm{MHz}, \mathrm{CDCl}_{3}\right), \delta[\mathrm{ppm}]$ : $170.10\left(\mathrm{COCH}_{3}\right), 166.43(\mathrm{C}(\mathrm{O}) \mathrm{O}), 165.71(\mathrm{C}(\mathrm{O}) \mathrm{O}), 133.78(\mathrm{ArC}), 128.75(\mathrm{ArC}), 128.70(\mathrm{ArC})$, 128.69 (ArC), $76.83(\mathrm{HNCCO}), 67.21\left(\mathrm{CNO}_{2}\right), 63.56\left(\mathrm{CH}_{2} \mathrm{CH}_{3}\right), 62.75\left(\mathrm{CH}_{2} \mathrm{CH}_{3}\right)$, $48.30\left(\mathrm{PhCCNO}_{2}\right), 22.97\left(\mathrm{COCH}_{3}\right), 13.84\left(\mathrm{CH}_{2} \mathrm{CH}_{3}\right), 13.76\left(\mathrm{CH}_{2} \mathrm{CH}_{3}\right)$.

HRMS calculated for $\mathrm{C}_{17} \mathrm{H}_{22} \mathrm{~N}_{2} \mathrm{O}_{7} 366.1727$, found 366.1728 . 
3.3.4. Synthesis of $(R)$-Diethyl 2,2-Dicyano-3-phenylcyclopropane-1,1-dicarboxylate (19) via MIRC Reaction

Benzylidenemalononitrile $(1.0 \mathrm{mmol})$, diethyl bromomalonate $(1.5 \mathrm{mmol})$, and the appropriate crown ether $(10 \mathrm{~mol} \%)$ were dissolved in anhydrous $\mathrm{CH}_{2} \mathrm{Cl}_{2}(3 \mathrm{~mL})$, and dry $\mathrm{Na}_{2} \mathrm{CO}_{3}(2.0 \mathrm{mmol})$ was added. The reaction mixture was stirred at room temperature. After completing the reaction, the mixture was filtered, then the organic phase was washed with $10 \%$ aq. $\mathrm{HCl}(3 \times 10 \mathrm{~mL})$ and then with water $(10 \mathrm{~mL})$, dried $\left(\mathrm{Na}_{2} \mathrm{CO}_{3}\right.$ and $\left.\mathrm{Na}_{2} \mathrm{SO}_{4}\right)$ and concentrated. The crude product was purified by preparative TLC using hexane-ethyl acetate (5:1) as the eluent to give a yellow oil. For the respective yields and ee values, see Table 4. Chiral HPLC: Kromasil 5-Amycoat ${ }^{\circledR}$ column, hexane:EtOH 85:15, major enantiomer $\mathrm{t}_{\mathrm{R}}=8.7 \mathrm{~min}$, minor enantiomer $\mathrm{t}_{\mathrm{R}}=7.4 \mathrm{~min} .[\alpha]_{D}^{22}=-20.3\left(c=1, \mathrm{CHCl}_{3}\right)(99 \%$ ee $)$.

${ }^{1} \mathrm{H}$ NMR (300 MHz, $\left.\mathrm{CDCl}_{3}\right), \delta(\mathrm{ppm}): 7.45-7.35(\mathrm{~m}, 5 \mathrm{H}, \operatorname{ArH}), 4.43(\mathrm{q}, J=7.2 \mathrm{~Hz}, 2 \mathrm{H}$, $\left.\mathrm{OCH}_{2}\right), 4.30-4.18\left(\mathrm{~m}, 2 \mathrm{H}, \mathrm{OCH}_{2}\right), 3.96(\mathrm{~s}, 1 \mathrm{H}, \mathrm{ArCH}), 1.39\left(\mathrm{t}, J=7.2 \mathrm{~Hz}, 3 \mathrm{H}, \mathrm{CH}_{2} \mathrm{CH}_{3}\right)$, $1.19\left(\mathrm{t}, J=7.2 \mathrm{~Hz}, 3 \mathrm{H}, \mathrm{CH}_{2} \mathrm{CH}_{3}\right) ;{ }^{13} \mathrm{C} \mathrm{NMR}\left(75 \mathrm{MHz}, \mathrm{CDCl}_{3}\right), \delta(\mathrm{ppm}): 163.05\left(\mathrm{COOC}_{2} \mathrm{H}_{5}\right)$, $161.06\left(\mathrm{COOC}_{2} \mathrm{H}_{5}\right), 129.67(\operatorname{ArC}), 129.10(\operatorname{ArC}), 128.76(\operatorname{ArC}), 127.31(\operatorname{ArC}), 111.86(\mathrm{CN})$, $109.71(\mathrm{CN}), 64.50\left(\mathrm{CH}_{2} \mathrm{CH}_{3}\right), 63.62\left(\mathrm{CH}_{2} \mathrm{CH}_{3}\right), 46.39(\mathrm{OCCCO}), 40.08(\mathrm{PhCH}), 16.32(\mathrm{NCCCN})$, $13.97\left(\mathrm{CH}_{2} \mathrm{CH}_{3}\right), 13.60\left(\mathrm{CH}_{2} \mathrm{CH}_{3}\right)$.

HRMS calculated for $\mathrm{C}_{17} \mathrm{H}_{16} \mathrm{~N}_{2} \mathrm{O}_{4} 312.1110$ found 312.1112.

\section{Conclusions}

New chiral crown ethers annulated to methyl 4,6-di-O-ethyl- $\alpha$-D-glucopyranoside $(\mathbf{1 a}-\mathbf{c})$ have been synthesized and tested in asymmetric reactions as phase transfer catalysts. Their effectiveness was compared to their 4,6-O-benzylidene analogues $(\mathbf{2 a}-\mathbf{c})$. It was found that the absence of the two-ring annulation affects the enantioselectivity rather negatively. Still, the results suggest that the effects of the protecting group(s) attached to the oxygen atoms in positions 4 and 6 of the carbohydrate and that of the sidearm are not independent of each other.

In the liquid-liquid model reactions, lariat ethers with a hydroxypropyl side chain were the most effective as observed to date. In the case of Michael addition of diethyl acetamidomalonate, the same phenomenon was experienced, which suggests that the interaction of the $\mathrm{OH}$ group has a crucial role in the formation of enantioselectivity. However, in the MIRC reaction of benzylidenemalononitrile and diethyl bromomalonate, the methoxypropyl side arm proved to be more effective. In addition, the 4,6-di-O-ethyl- $\alpha$-Dglucopyranoside-based crown catalyst (1b) was superior to its 4,6-O-benzylidene analogue (2b) in this cyclopropanation reaction. In this case, better flexibility was beneficial to the asymmetric induction.

Since the new 4,6-di-O-ethyl-glucoside-based crown ethers do not contain acidsensitive groups, they may be suitable for recovery through salt formation by extraction with mineral acid without any kind of structural alteration. By changing the 4,6 protecting groups of the glucose unit, lipophilicity and thus recoverability can be affected. Attempts to recover and reuse this type of chiral macrocycles are ongoing in our research group.

Author Contributions: Supervision, P.B. (Péter Bakó) and Z.R.; Investigation, I.O., B.V., L.H. and P.B. (Péter Bagi); Resources: L.H., P.B. (Péter Bagi), Z.R. and P.B. (Péter Bakó). All authors have read and agreed to the published version of the manuscript.

Funding: This research received no external funding.

Conflicts of Interest: The authors declare no conflict of interest.

Sample Availability: Samples of the compounds are not available from the authors.

\section{References}

1. List, B.; Lerner, A.R.A.; Barbas, C.F. Proline-Catalyzed Direct Asymmetric Aldol Reactions. J. Am. Chem. Soc. 2000, 122, $2395-2396$. [CrossRef]

2. Ahrendt, K.A.; Borths, C.J.; MacMillan, D.W.C. New Strategies for Organic Catalysis: The First Highly Enantioselective Organocatalytic Diels-Alder Reaction. J. Am. Chem. Soc. 2000, 122, 4243-4244. [CrossRef] 
3. Berkessel, A.; Gröger, H. Asymmetric Organocatalysis, 1st ed.; Wiley-VCH: Weinheim, Germany, 2005. [CrossRef]

4. Macmillan, D.W.C. The advent and development of organocatalysis. Nature 2008, 455, 304-308. [CrossRef] [PubMed]

5. Kacprzak, K.; Gawronski, J. Cinchona Alkaloids and Their Derivatives: Versatile Catalysts and Ligands in Asymmetric Synthesis. Synthesis 2001, 961-998.

6. McCooey, S.H.; Connon, S.J. Urea- and Thiourea-Substituted Cinchona Alkaloid Derivatives as Highly Efficient Bifunctional Organocatalysts for the Asymmetric Addition of Malonate to Nitroalkenes: Inversion of Configuration at C9 Dramatically Improves Catalyst Performance. Angew. Chem. Int. Ed. 2005, 44, 6367-6370. [CrossRef]

7. Vakulya, B.; Varga, S.; Csámpai, A.; Soós, T. Highly Enantioselective Conjugate Addition of Nitromethane to Chalcones Using Bifunctional Cinchona Organocatalysts. Org. Lett. 2005, 7, 1967-1969. [CrossRef] [PubMed]

8. Hamza, A.; Schubert, G.; Soós, A.T.; Pápai, I. Theoretical Studies on the Bifunctionality of Chiral Thiourea-Based Organocatalysts: Competing Routes to C-C Bond Formation. J. Am. Chem. Soc. 2006, 128, 13151-13160. [CrossRef]

9. Bartoli, G.; Bosco, M.; Carlone, A.; Cavalli, A.; Locatelli, M.; Mazzanti, A.; Ricci, P.; Sambri, L.; Melchiorre, P. Organocatalytic Asymmetric Conjugate Addition of 1,3-Dicarbonyl Compounds to Maleimides. Angew. Chem. Int. Ed. 2006, 45, 4966-4970. [CrossRef]

10. Malerich, J.P.; Hagihara, K.; Rawal, V.H. Chiral Squaramide Derivatives are Excellent Hydrogen Bond Donor Catalysts. J. Am. Chem. Soc. 2008, 130, 14416-14417. [CrossRef]

11. Kótai, B.; Kardos, G.; Hamza, A.; Farkas, V.; Pápai, I.; Soós, T. On the Mechanism of Bifunctional Squaramide-Catalyzed Organocatalytic Michael Addition: A Protonated Catalyst as an Oxyanion Hole. Chem. Eur. J. 2014, 20, 5631-5639. [CrossRef] [PubMed]

12. Varga, E.; Mika, L.T.; Csámpai, A.; Holczbauer, T.; Kardos, G.; Soós, T. Mechanistic investigations of a bifunctional squaramide organocatalyst in asymmetric Michael reaction and observation of stereoselective retro-Michael reaction. RSC Adv. 2015, 5, 95079-95086. [CrossRef]

13. Grayson, M.N. Mechanism and Origins of Stereoselectivity in the Cinchona Thiourea- and Squaramide-Catalyzed Asymmetric Michael Addition of Nitroalkanes to Enones. J. Org. Chem. 2017, 82, 4396-4401. [CrossRef] [PubMed]

14. Boratynski, P.J.; Zielinska-Błajet, M.; Skarżewski, J. Chapter Two-Cinchona Alkaloids—Derivatives and Applications. In The Alkaloids, 1st ed.; Knölker, H.-J., Ed.; Academic Press: Cambridge, MA, USA, 2019; pp. 29-145.

15. Dehmlow, E.V.; Dehmlow, S.S. Phase Transfer Catalysis, 3rd ed.; VCH: New York, NY, USA, 1993.

16. Starks, C.M.; Liotta, C.L.; Halpern, M.E. Phase Transfer Catalysis: Fundamentals, Applications, and Industrial Perspectives; Chapman \& Hall: New York, NY, USA, 1994.

17. O' Donnell, M.I. Asymmetric Phase Transfer Reactions. In Catalytic Asymmetric Synthesis, 2nd ed.; Ojima, I., Ed.; Wiley-VCH: New York, NY, USA, 2000; pp. 727-745.

18. Ooi, T.; Maruoka, K. Recent Advances in Asymmetric Phase-Transfer Catalysis. Angew. Chem. Int. Ed. 2007, 46, 4222-4266. [CrossRef] [PubMed]

19. Marouka, K. Asymmetric Phase Transfer Catalysis; Wiley-VCH: Weinheim, Germany, 2008.

20. Shirakawa, S.; Maruoka, K. Recent Developments in Asymmetric Phase-Transfer Reactions. Angew. Chem. Int. Ed. 2013, 52, 4312-4348. [CrossRef] [PubMed]

21. Jayaraman, S.; Kumaraguru, D.; Arockiam, J.B.; Paulpandian, S.; Rajendiran, B.; Siva, A. Highly enantioselective asymmetric Michael addition reactions with new chiral multisite phase-transfer catalysts. Synlett 2014, 25, 1685-1691. [CrossRef]

22. Kaneko, S.; Kumatabara, Y.; Shirakawa, S. A new generation of chiral phase-transfer catalysts. Org. Biomol. Chem. 2016, 14, 5367-5376. [CrossRef]

23. Schettini, R.; De Riccardis, F.; Della Sala, G.; Izzo, I. Enantioselective Alkylation of Amino Acid Derivatives Promoted by Cyclic Peptoids under Phase-Transfer Conditions. J. Org. Chem. 2016, 81, 2494-2505. [CrossRef] [PubMed]

24. Schörgenhumer, J.; Tiffner, M.; Waser, M. Chiral phase-transfer catalysis in the asymmetric $\alpha$-heterofunctionalization of prochiral nucleophiles. Beilstein J. Org. Chem. 2017, 13, 1753-1769. [CrossRef]

25. Schettini, R.; Sicignano, M.; De Riccardis, F.; Izzo, I.; Della Sala, G. Macrocyclic Hosts in Asymmetric Phase-Transfer Catalyzed Reactions. Synthesis 2018, 50, 4777-4795. [CrossRef]

26. Zhang, J.; Zhao, G. Enantioselective Mannich reaction of $\gamma$-malonate-substituted $\alpha, \beta$-unsaturated esters with N-Boc imines catalyzed by chiral bifunctional thiourea-phosphonium salts. Tetrahedron 2019, 75, 1697-1705. [CrossRef]

27. Mahajan, D.P.; Godbole, H.M.; Singh, G.P.; Shenoy, G.G. Synthesis of novel phase transfer catalysts derived from proline-mandelic acid/tartaric acid: Their evaluation in enantioselective epoxidation and Darzens condensation. J. Chem. Sci. 2019, 131, 22. [CrossRef]

28. Pan, J.; Wu, J.-H.; Zhang, H.; Ren, X.; Tan, J.-P.; Zhu, L.; Zhang, H.-S.; Jiang, C.; Wang, T. Highly Enantioselective Synthesis of Fused Tri- and Tetrasubstituted Aziridines: Aza-Darzens Reaction of Cyclic Imines with $\alpha$-Halogenated Ketones Catalyzed by Bifunctional Phosphonium Salt. Angew. Chem. Int. Ed. 2019, 58, 7425-7430. [CrossRef] [PubMed]

29. Maruoka, K. Design of high-performance chiral phase-transfer catalysts with privileged structures. Proc. Jpn. Acad. Ser. B Phys. Biol. Sci. 2019, 95, 1-16. [CrossRef]

30. Wang, H. Chiral Phase-Transfer Catalysts with Hydrogen Bond: A Powerful Tool in the Asymmetric Synthesis. Catalysts 2019, 9 , 244. [CrossRef] 
31. Majdecki, M.; Tyszka-Gumkowska, A.; Jurczak, J. Highly Enantioselective Epoxidation of $\alpha$, $\beta$-unsaturated ketones using amide-based cinchona alkaloids as hybrid phase-transfer catalysts. Org. Lett. 2020, 22, 8687-8691. [CrossRef]

32. Lu, D.; Liu, X.; Wu, J.-H.; Zhang, S.; Tan, J.-P.; Yu, X.; Wang, T. Asymmetric Construction of Bispiro-Cyclopropane-Pyrazolones via a [2+1] Cyclization Reaction by Dipeptide-Based Phosphonium Salt Catalysis. Adv. Synth. Catal. 2020, 362, 1966-1971. [CrossRef]

33. Tian, Z.; Meng, X.; Luo, Y.; Cao, S.; Zhao, G. A novel quaternary ammonium salts derived from $\alpha$-amino acids with large steric hindrance group and its application in asymmetric Mannich reaction. Tetrahedron 2020, 76, 131484. [CrossRef]

34. Genov, G.R.; Douthwaite, J.L.; Lahdenperä, A.S.K.; Gibson, D.C.; Phipps, R.J. Enantioselective remote C-H activation directed by a chiral cation. Science 2020, 367, 1246-1251. [CrossRef]

35. Sabah, K.J.; Zahid, N.I.; Hashim, R. Synthesis of new chiral macrocycles-based glycolipids and its application in asymmetric Michael addition. Res. Chem. Intermed. 2021, 47, 2653-2667. [CrossRef]

36. Cram, D.J.; Sogah, G.D.Y. Chiral Crown Complexes Catalyze Michael Addition Reactions to Give Adducts in High Optical Yields. J. Chem. Soc. Chem. Commun. 1981, 13, 625-628. [CrossRef]

37. Stoddart, J.F. Synthetic chiral receptor molecules from natural products. In Progress in Macrocyclic Chemistry; Izatt, R.M., Christensen, J.J., Eds.; Wiley-Interscience: New York, NY, USA, 1981; Volume 2, pp. 173-250.

38. Stoddart, J.F. Chiral crown ethers. In Topics in Stereochemistry; Eliel, E.L., Wielen, S.H., Eds.; Wiley: New York, NY, USA, 1988; Volume 17, pp. 207-288.

39. Jarosz, S.; Listkowski, A. Sugar derived crown ethers and their analogs: Synthesis and properties. Curr. Org. Chem. 2006, 10, 643-662. [CrossRef]

40. Miethchen, R.; Fehring, V. Chirale Kronenether mit integriertem, 1,4-verbrückten D-Glucopyranose-Baustein. Synthesis 1998, 1, 94-98. [CrossRef]

41. Bakó, P.; Tôke, L. Novel monoaza- and diazacrown ethers incorporating sugar units and their extraction ability towards picrate salts. J. Incl. Phenom. Macrocycl. Chem. 1995, 23, 195-201. [CrossRef]

42. Wenzel, T.J.; Thurston, J.E.; Sek, D.C.; Joly, J.-P. Utility of crown ethers derived from methyl $\beta$-D-galactopyranoside and their lathanide couples as chiral NMR discriminating agents. Tetrahedron Asymmetry 2001, 12, 1125-1130. [CrossRef]

43. Miethchen, R.; Faltin, F.; Fehring, V. Chiral Crown Ethers Based on Galactopyranosides. Synthesis 2002, 2002, 1851-1856. [CrossRef]

44. Pietraszkiewicz, M.; Salanski, P.; Jurczak, J. Synthesis of novel chiral [2.2.1]cryptands incorporating sugars. Tetrahedron 1984, 40, 2971-2973. [CrossRef]

45. Bakó, P.; Mako, A.; Keglevich, G.; Kubinyi, M.; Pál, K. Synthesis of d-mannose-based azacrown ethers and their application in enantioselective reactions. Tetrahedron Asymmetry 2005, 16, 1861-1871. [CrossRef]

46. Mako, A.; Menyhárd, D.K.; Bakó, P.; Keglevich, G.; Tôke, L. Theoretical study of the asymmetric phase-transfer mediated epoxidation of chalcone catalyzed by chiral crown ethers derived from monosaccharides. J. Mol. Struct. 2008, 892, 336-342. [CrossRef]

47. Van Maarschalkerwaart, D.A.H.; Willard, N.P.; Pandit, U.K. Synthesis of carbohydrate containing crown ethers and their application as catalysts in asymmetric Michael additions. Tetrahedron 1992, 48, 8825-8840. [CrossRef]

48. Sharma, G.V.M.; Reddy, V.G.; Krishna, P.R. Synthesis of new chiral 18-crown-6 ethers from D-xylose. Tetrahedron Asymmetry 1999, 10, 3777-3784. [CrossRef]

49. Szabó, T.; Rapi, Z.; Keglevich, G.; Szöllősy, Á.; Drahos, L.; Bakó, P. Synthesis of L-arabinose-based crown ethers and their applications as enantioselective phase transfer catalysts. Arkivoc 2012, 2012, 36-48. [CrossRef]

50. Joly, J.-P.; Nazhaoui, M.; Dumont, B. Synthesis and complexation behaviour of some crown ethers derived from D-hexopyranosides and D-hexopyranosides and D-mannitol towards racemic phenylglycine salts. Bull. Soc. Chim. Fr. 1994, 131, 369-380.

51. Gryko, D.T.; Piatek, P.; Jurczak, J. An Efficient Method for Preparation of Chiral Macrocyclic Bisamides Starting from Diol Derivatives of D-Mannitol and L-Tartaric Acid. Synthesis 1999, 336-340. [CrossRef]

52. Nemcsok, T.; Rapi, Z.; Keglevich, G.; Grün, A.; Bakó, P. Synthesis of D-mannitol-based crown ethers and their application as catalysts in asymmetric phase transfer reactions. Chirality 2018, 30, 407-419. [CrossRef]

53. Rapi, Z.; Nemcsok, T.; Pálvölgyi, Á.; Keglevich, G.; Grün, A.; Bakó, P. Synthesis of L-threitol-based crown ethers and their application as enantioselective phase transfer catalyst in Michael additions. Chirality 2017, 29, 257-272. [CrossRef] [PubMed]

54. Bakó, P.; Keglevich, G.; Rapi, Z. Asymmetric phase transfer reactions catalyzed by chiral crown ethers derived from monosaccharides. Lett. Org. Chem. 2010, 7, 645-656. [CrossRef]

55. Orbán, I.; Bakó, P.; Rapi, Z. Carbohydrate-Based Azacrown Ethers in Asymmetric Syntheses. Chemistry 2021, 3, 550-577. [CrossRef]

56. Rapi, Z.; Bakó, P.; Drahos, L.; Keglevich, G. Side-Arm Effect of a Methyl $\alpha$-D-Glucopyranoside Based Lariat Ether Catalysts in Asymmetric Syntheses. Heteroatom Chem. 2015, 26, 63-71. [CrossRef]

57. Pálvölgyi, M.; Rapi, Z.; Ozohanics, O.; Toth, G.; Keglevich, G.; Bakó, P. Synthesis of alkyl $\alpha$ - and $\beta$-D-glucopyranoside-based chiral crown ethers and their application as enantioselective phase-transfer catalysts. Res. Chem. Intermed. 2017, 44, 1627-1645. [CrossRef]

58. Rapi, Z.; Nemcsok, T.; Bagi, P.; Keglevich, G.; Bakó, P. Synthesis of chiral crown ethers derived from d-galactose and their application in enantioselective reactions. Tetrahedron 2019, 75, 3993-4004. [CrossRef] 
59. Nemcsok, T.; Rapi, Z.; Bagi, P.; Hou, G.Y.; Orbán, I.; Keglevich, G.; Bakó, P. Enantioselective cyclopropanation of conjugated cyanosulfones using carbohydrate-based crown ether catalysts. Tetrahedron 2020, 76, 130965. [CrossRef]

60. Bakó, P.; Kiss, T.; Tőke, L. Chiral azacrown ethers derived from D-glucose as catalysts for enantioselective Michael addition. Tetrahedron Lett. 1997, 38, 7259-7262. [CrossRef]

61. Makó, A.; Szöllősy, Á.; Keglevich, G.; Menyhárd, D.K.; Bakó, P.; Tőke, L. Synthesis of methyl- $\alpha$-D-glucopyranoside-based azacrown ethers and their application in enantioselective reactions. Monats. Chem. 2008, 139, 525-535. [CrossRef]

62. Bakó, P.; Bajor, Z.; Tőke, L.J. Synthesis of novel chiral crown ethers derived from D-glucose and their application to an enantioselective Michael reaction. Chem. Soc. Perkin Trans. I 1999, 24, 3651-3655. [CrossRef]

63. Bakó, P.; Czinege, E.; Bakó, T.; Czugler, M.; Tőke, L. Asymmetric C-C bond forming reactions with chiral crown catalysts derived from d-glucose and d-galactose. Tetrahedron Asymmetry 1999, 10, 4539-4551. [CrossRef]

64. Bakó, P.; Bakó, T.; Mészáros, A.; Keglevich, G.; Szöllősy, Á.; Bodor, S.; Makó, A.; Tőke, L. Phase Transfer Catalysed Asym-metric Epoxidation of Chalcones Using Chiral Crown Ethers Derived from D-Glucose and D-Mannose. Synlett 2004, 2004, 643-646. [CrossRef]

65. Rapi, Z.; Démuth, B.; Keglevich, G.; Grün, A.; Drahos, L.; Sóti, P.L.; Bakó, P. Enantioselective Michael addition of malonates to aromatic nitroalkenes catalyzed by monosaccharide-based chiral crown ethers. Tetrahedron Asymmetry 2014, 25, 141-147. [CrossRef]

66. Bakó, P.; Rapi, Z.; Grün, A.; Nemcsok, T.; Hegedûs, L.; Keglevich, G. Asymmetric Michael addition of malonates to enones catalyzed by an $\alpha$-D-glucopyranoside-based brown ether. Synlett 2015, 26, 1847-1851. [CrossRef]

67. Whistler, R.L.; Wolform, M.L. Methods in Carbohydrate Chemistry; Academic Press Inc.: New York, NY, USA, 1963; Volume 2, pp. 307-308.

68. Lonnecker, A.T.; Lim, Y.H.; Felder, S.E.; Besset, C.J.; Wooley, K.L. Four Different Regioisomeric Polycarbonates Derived from One Natural Product, d-Glucose. Macromolecules 2016, 49, 7857-7867. [CrossRef]

69. Deng, S.; Gangadharmath, U.; Chang, C.T. Sonochemistry: A Powerful Way of Enhancing the Efficiency of Carbohydrate Synthesis. J. Org. Chem. 2006, 71, 5179-5185. [CrossRef] [PubMed]

70. Bakó, P.; Szöllősy, Á.; Bombicz, P.; Tőke, L. Asymmetric C-C Bond Forming Reactions by Chiral Crown Catalysts; Darzens Condensation and Nitroalkane Addition to the Double Bond. Synlett 1997, 291-292. [CrossRef]

71. Bakó, P.; Rapi, Z.; Keglevich, G.; Szabó, T.; Soti, P.L.; Vigh, T.; Grun, A.; Holczbauer, T. Asymmetric C-C bond formation via Darzens condensation and Michael addition using monosaccharide-based chiral crown ethers. Tetrahedron Lett. 2011, 52, 1473-1476. [CrossRef]

72. Rapi, Z.; Nemcsok, T.; Grün, A.; Pálvölgyi, Á.; Samu, G.; Hessz, D.; Kubinyi, M.; Kállay, M.; Keglevich, G.; Bakó, P. Asymmetric cyclopropanation reactions catalyzed by carbohydrate-based crown ethers. Tetrahedron 2018, 74, 3512-3526. [CrossRef] 